\title{
ROSENTHAL'S THEOREM FOR SUBSPACES OF NONCOMMUTATIVE $L_{p}$
}

\author{
MARIUS JUNGE AND JAVIER PARCET
}

\begin{abstract}
We show that a reflexive subspace of the predual of a von Neumann algebra embeds into a noncommutative $L_{p}$ space for some $p>1$. This is a noncommutative version of Rosenthal's result for commutative $L_{p}$ spaces. Similarly for $1 \leq q<2$, an infinite dimensional subspace $X$ of a noncommutative $L_{q}$ space either contains $\ell_{q}$ or embeds in $L_{p}$ for some $q<p<2$. The novelty in the noncommutative setting is a double sided change of density.
\end{abstract}

\section{INTRODUCTION}

The theory of noncommutative $L_{p}$ spaces has a long tradition in Banach space theory and the theory of operator algebras [GK69, Haa79, Hil81, TJ84, Fac87] and provides the background for recent progress in noncommutative analysis and probability [PX97, JLX03, JX03]. In the commutative setting, the work of Kadec-Pelczyński [KP62] and Rosenthal [Ros73] on subspaces of $L_{p}$ are corner stones for the understanding of general Banach space properties. In this paper we prove the noncommutative version of Rosenthal's result.

Theorem (Rosenthal '73). A reflexive subspace of $L_{1}$ embeds into $L_{p}$ for some $p>1$.

The problem of generalizing Rosenthal theorem to the noncommutative setting is open for at least 20 years. This problem has an interesting history. In his seminal paper [Pis86b] on factorization properties, Pisier described a new approach to some factorization results by Maurey obtained from Nikishin's theorem. In this paper Pisier comes very close to proving the noncommutative version of Rosenthal's result. Indeed, he shows that a reflexive subspace of a von Neumann algebra predual embeds into an interpolation space between an $L_{1}$ space and certain (unusual) $L_{2}$ space (see below). Since then it has been a mystery how to modify the argument and to obtain a subspace of a noncommutative $L_{p}$ space. Noncommutative $L_{p}$ spaces have been defined by Dixmier, Kunze and Segal in the semifinite setting (see also Nelson [Nel74]) and by Haagerup [Haa79] in the non-tracial case (see also [Hil81] for Connes' approach). Randrianantoanina [Ran02] has an argument in the semifinite setting which is different from ours and does not provide a good control of the constants. In this paper we use modular theory of operator algebras in conjunction with a noncommutative version of the Peter Jones theorem due to Pisier [Pis92] (related to estimates of Kaftal, Larsen and Weiss [KLW92] for triangular matrices) to solve the problem: 
Theorem A. Let $N$ be a von Neumann algebra. A reflexive subspace of $L_{1}(N)$ embeds into $L_{p}(N)$ for some $p>1$.

The new interesting point in our proof is the natural change of density argument. We show that there exists a positive density $d \in L_{1}(N)$ such that $\operatorname{tr}(d)=1$ and a mapping $u: X \rightarrow L_{p}(N)$ such that

$$
x=d^{1-\frac{1}{p}} u(x)+u(x) d^{1-\frac{1}{p}} .
$$

In the $\sigma$-finite case this completely determines $u$. For simplicity let us assume that $N$ is finite and $d=\sum_{j} d_{j} e_{j}$ has a countable spectrum. Then the map $u$ is given by the following relation

$$
u(x)=\sum_{i, j}\left(d_{i}^{1-\frac{1}{p}}+d_{j}^{1-\frac{1}{p}}\right)^{-1} e_{i} x e_{j} .
$$

Pisier's approach to this result [Pis86b] is used as a starting point in our proof. For subspaces of $L_{q}(N)$ with $q>1$ we have a similar result, which extends the most general form of Rosenthal's theorem [Ros73, Theorem 8] to the noncommutative setting.

Theorem B. Let $N$ be a von Neumann algebra and fix $1 \leq q<2$. Given a subspace $X$ of $L_{q}(N)$ not containing $\ell_{q}$, there exists a positive density $d \in L_{1}(N)$ with $\operatorname{tr}(d)=1$ and a map $u: X \rightarrow L_{p}(N)$ for some index $q<p<2$ such that

$$
x=d^{\frac{1}{q}-\frac{1}{p}} u(x)+u(x) d^{\frac{1}{q}-\frac{1}{p}} .
$$

In particular, the space $X$ embeds isomorphically into $L_{p}(N)$.

This result, which also works for linear maps, is closely related to Grothendieck type inequalities by Lust-Piquard, see [LP92] and [LPX]. One of the main obstacles in our approach to Theorem B is that the technique of noncommutative maximal functions is not well-enough understood for proving Nikishin type results. Therefore we have to work in the dual setting. Pisier's arguments for $q>1$ are genuinely very different from the case $q=1$ which, by duality, leads to linear maps on $C^{*}$-algebras. A common characteristic of Pisier's factorization results in Pis86b] is a certain differentiation argument. This is our motivation for the following new inequality. Let $2 \leq p<\infty$ and $a, x$ be positive elements in $L_{p}(N)$. Then we have

$$
\|a+x\|_{p}^{p} \leq\|a\|_{p}^{p}+p 2^{p-1} \max \left\{\left\|a^{p-1} x\right\|_{1},\|x\|_{p}^{p}\right\} .
$$

In the commutative case the triangle inequality in $L_{p-1}$ provides a similar estimate with $2^{p}-1$ instead of $p 2^{p-1}$. For $2 \leq p \leq 3$ operator convexity of $t \mapsto t^{p-1}$ provides an even better estimate. Combined with ultraproduct techniques, the differential inequality (11) is a substitute for some of Pisier's arguments in [Pis86b]. 
Another technical difficulty concerns complex interpolation of intersections. We refer to [JP05] for many results in this direction. For a long time, our hope has been to use free probability to show that interpolation and intersection commute in this particular setting. However, at the time of this writing some aspects of harmonic analysis are yet to be discovered before this approach might be successful. In the Banach space setting of this paper, we may use different tools from harmonic analysis. Let us be more specific. We consider a normal faithful state $\phi(\cdot)=\operatorname{tr}(d \cdot)$ on a von Neumann algebra $N$ and Pisier's symmetric norm

$$
\|x\|_{\Delta_{2}(\phi)}=\left(\phi\left(x x^{*}\right)+\phi\left(x^{*} x\right)\right)^{\frac{1}{2}} \sim \max \left\{\left\|d^{\frac{1}{2}} x\right\|_{2},\left\|x d^{\frac{1}{2}}\right\|_{2}\right\} .
$$

We will show that

$$
\|x\|_{\left[N, \Delta_{2}(\phi)\right]_{\frac{2}{p}}} \leq c(p) \max \left\{\left\|d^{\frac{1}{p}} x\right\|_{p},\left\|x d^{\frac{1}{p}}\right\|_{p}\right\}
$$

holds for all $x \in N$ and $2 \leq p<\infty$. We can show that the orthogonal projection from $L_{2}(N \oplus N)$ to $\Delta_{2}(\phi)$ extends to a bounded operator for other values of $p$. This allows us to construct the map $u$ in Theorem $\mathrm{A}$.

In combination with the results from [JR], we obtain some applications to the theory of subsymmetric sequences. A sequence $\left(x_{n}\right)$ in a Banach space $X$ is called subsymmetric if there exists a constant $c$ such that

$$
\left\|\sum_{n} a_{n} x_{n}\right\|_{X} \sim_{c}\left\|\sum_{n} a_{n} x_{k_{n}}\right\|_{X}
$$

holds for every strictly increasing sequence $\left(k_{n}\right)$ and arbitrary coefficients $\left(a_{n}\right)$. We refer to the work of Aldous [Ald81] and Krivine-Maurey [KM81 for the fact that commutative $L_{p}$ spaces are stable. This implies in particular that subsymmetric sequences are symmetric, i.e. we may replace subsequences $\left(k_{n}\right)$ by arbitrary permutation $(\sigma(n))$. However, due to a result by Marcolino Nhany [MN97], noncommutative $L_{p}$ spaces are in general not stable.

Corollary C. If $\left(x_{n}\right) \subset N_{*}$ is a subsymmetric sequence, then $\left(x_{n}\right)$ is either symmetric or the space $X=\operatorname{span}\left\{x_{n} \mid n \geq 1\right\}$ contains $\ell_{1}$. In particular, $X$ always contains a symmetric subspace.

The paper is organized as follows. In section 1 we prove (2) and the interpolation results for intersections based on the Peter Jones theorem. This allows us to prove Theorem A and Corollary C in section 2. Inequality (1) and Theorem B are proved in the last section of the paper. We use standard notation from the theory of operator algebras [Tak79, KR97a, KR97b] and the theory of noncommutative $L_{p}$ spaces [Ter81] (see also [Ter82]). The reader is assumed to be familiar with basic ingredients of modular theory and the definition of Haagerup's noncommutative $L_{p}$ spaces, see [JX03, PX03] for relevant definitions. However, the main ideas can be understood by 'thinking semifinite'. 


\section{An interpolation Result}

In this section we provide the main new interpolation results on intersections and, in particular, the key inequality (2) will be obtained. In this paper we will use Haagerup's definition of noncommutative $L_{p}$ spaces. Indeed, one first considers the crossed product $M=N \rtimes_{\sigma_{t}^{\phi}} \mathbb{R}$ with respect to a normal semifinite faithful weight $\phi$ on $N$. Then $M$ is semifinite and there exists a unique normal semifinite faithful trace $\tau$ on $M$ such that the dual action $\theta_{s}: M \rightarrow M$ satisfies $\tau\left(\theta_{s}(d)\right)=e^{-s} \tau(d)$ for all $s \in \mathbb{R}$. Haagerup's $L_{p}$ space is defined as follows

$$
L_{p}(N)=\left\{x \in L_{0}(M, \tau) \mid \theta_{s}(x)=e^{-\frac{s}{p}} x\right\},
$$

where $L_{0}(M, \tau)$ stands for the space of $\tau$-measurable operators affiliated to $M$. For $p=\infty$ we see that $L_{\infty}(N)=N$ is the set of $\theta_{s}$-invariant operators. Let us note that the polar decomposition $x=u|x|$ for $x \in L_{p}(N)$ satisfies $u \in N$ and $|x| \in L_{p}(N)$. We refer to [Ter81] for more details, see also [JX03, PX03, Tak03a, Tak03b]. An important feature of the Haagerup $L_{1}(N)$ space is the distinguished linear functional $\operatorname{tr}: L_{1}(N) \rightarrow \mathbb{C}$. This linear map implements the isomorphism between $L_{1}(N)$ and $N_{*}$. More precisely, for every normal functional $\phi \in N_{*}$ there exists a unique density $d_{\phi}$ with $\phi(x)=\operatorname{tr}\left(d_{\phi} x\right)$. Moreover, given $1 \leq p<\infty$, the trace functional $\operatorname{tr}$ also implements the duality between $L_{p}(N)$ and $L_{p^{\prime}}(N)$. That is, $L_{p}(N)^{*}$ is exactly the space of linear functionals $\phi(x)=\operatorname{tr}(d x)$ with $d \in L_{p^{\prime}}(N)$ and $\frac{1}{p}+\frac{1}{p^{\prime}}=1$. The norm in $L_{p}(N)$ is given by

$$
\|x\|_{p}=\operatorname{tr}\left(|x|^{p}\right)^{\frac{1}{p}} .
$$

We also have Hölder's inequality $\|x y\|_{p} \leq\|x\|_{q}\|y\|_{r}$ whenever $\frac{1}{p}=\frac{1}{q}+\frac{1}{r}$.

The drawback of Haagerup's construction is the unfamiliar situation that for $p \neq q$ we have $L_{p}(N) \cap L_{q}(N)=\{0\}$. In particular, this implies that Haagerup $L_{p}$ spaces do not form an interpolation scale. However, in this paper interpolation techniques are important. We shall assume that the reader is familiar with the complex interpolation method. Let us briefly review Kosaki's results [Kos84a on interpolation of $L_{p}$ spaces which are crucial in our paper. Once and for all in the sequel, let us fix a von Neumann algebra $N$ equipped with a normal faithful state $\phi$ so that $\phi(x)=\operatorname{tr}(d x)$ is given by a positive density $d \in L_{1}(N)$. Then we may consider the injective maps

$$
\iota_{\eta}: x \in N \mapsto d^{1-\eta} x d^{\eta} \in L_{1}(N) \quad \text { for } \quad 0 \leq \eta \leq 1 .
$$

A little bit of modular theory is required to show that these maps are indeed injective, see [Kos84a, Jun04. Thus, for fixed $0 \leq \eta \leq 1,\left(A_{0}, A_{1}\right)=\left(\iota_{\eta}(N), L_{1}(N)\right)$ is an interpolation couple embedded in $L_{1}(N)$ as a topological vector space. To be very precise, we recall that $\|x\|_{A_{0}}=\left\|\iota_{\eta}^{-1}(x)\right\|_{N}$ and $\|x\|_{A_{1}}=\|x\|_{L_{1}(N)}$. In the literature, the choices $\eta=0, \frac{1}{2}, 1$ 
are the most important ones. Kosaki showed that

$$
\left[\iota_{\eta}(N), L_{1}(N)\right]_{\frac{1}{p}}=d^{\frac{1-\eta}{p^{\prime}}} L_{p}(N) d^{\frac{\eta}{p^{\prime}}}
$$

holds isometrically. This means exactly that

$$
\|x\|_{p}=\left\|d^{\frac{1-\eta}{p^{\prime}}} x d^{\frac{\eta}{p^{\prime}}}\right\|_{\left[\iota_{\eta}(N), L_{1}(N)\right]_{\frac{1}{p}}} \quad \text { for all } \quad x \in L_{p}(N) .
$$

If $1 \leq q<p \leq \infty$ and $\frac{1}{s}=\frac{1}{q}-\frac{1}{p}$, we may also consider the embedding

$$
\iota_{p, q, \eta}: x \in L_{p}(N) \mapsto d^{\frac{1-\eta}{s}} x d^{\frac{\eta}{s}} \in L_{q}(N) .
$$

Then, the reiteration theorem for complex interpolation immediately gives

$$
\left[\iota_{p, q, \eta}\left(L_{p}(N)\right), L_{q}(N)\right]_{\theta}=\iota_{r, q, \eta}\left(L_{r}(N)\right)
$$

for $\frac{1}{r}=\frac{1-\theta}{p}+\frac{\theta}{q}$. These interpolation results from Kos84a will be used freely in this text.

Our aim in this section is to prove a similar result for a double sided embedding with respect to a fixed density $d$ of a normal faithful state $\phi$. For $1 \leq q<p \leq \infty$ we define the following norms

$$
\|x\|_{L_{p, q}^{r}(\phi)}=\left\|d^{\frac{1}{q}-\frac{1}{p}} x\right\|_{q} \quad \text { and } \quad\|x\|_{L_{p, q}^{c}(\phi)}=\left\|x d^{\frac{1}{q}-\frac{1}{p}}\right\|_{q} .
$$

Let us write $L_{p, q}^{r}(\phi)$ and $L_{p, q}^{c}(\phi)$ for the respective closures of $L_{p}(N)$ with respect to the norms above. Here $r, c$ are chosen because similar expressions appear for square function inequalities in noncommutative martingale theory. They correspond to $\eta=0$ and $\eta=1$ in the context of Kosaki's embedding. We will work with the intersection

$$
\Delta_{p, q}(\phi)=L_{p, q}^{r}(\phi) \cap L_{p, q}^{c}(\phi),
$$

defined as the completion of $L_{p}(N)$ with respect to the norm

$$
\|x\|_{\Delta_{p, q}(\phi)}=\max \left\{\|x\|_{L_{p, q}^{r}(\phi)},\|x\|_{L_{p, q}^{c}(\phi)}\right\} .
$$

Of course, up to an absolute constant, we may replace the maximum above by the sum or any other $p$-sum. We might use this equivalence below. We also have a natural embedding

$$
j_{p, q}: \Delta_{p, q}(\phi) \rightarrow L_{q}(N) \oplus L_{q}(N), \quad j_{p, q}(x)=\left(d^{\frac{1}{q}-\frac{1}{p}} x, x d^{\frac{1}{q}-\frac{1}{p}}\right) .
$$

According to Hölder's inequality we have a contractive inclusion $L_{p}(N) \subset \Delta_{p, q}(\phi)$ given by the identity map. Therefore $\left(L_{p}(N), \Delta_{p, q}(\phi)\right)$ is an interpolation couple with dense intersection. When $p=\infty$ we shall write $\Delta_{q}(\phi)$ for $\Delta_{\infty, q}(\phi)$. Thus, for $(p, q)=(\infty, 2)$ we find the well-known Hilbert space already mentioned in the Introduction

$$
\|x\|_{\Delta_{2}(\phi)} \sim\left(\left\|d^{\frac{1}{2}} x\right\|_{2}^{2}+\left\|x d^{\frac{1}{2}}\right\|_{2}^{2}\right)^{\frac{1}{2}}=\phi\left(x x^{*}+x^{*} x\right)^{\frac{1}{2}}=\sqrt{2} \phi\left(|x|_{s}^{2}\right)^{\frac{1}{2}} .
$$


Here we followed Pisier's notation

$$
|x|_{s}=\sqrt{\frac{x^{*} x+x x^{*}}{2}}
$$

Our main result in this section is the following.

Theorem 1.1. Let $1 \leq q<p \leq \infty$ and $\frac{1}{r}=\frac{1-\theta}{p}+\frac{\theta}{q}$ for $0<\theta<1$. Then

a) We have an isomorphism

$$
\Delta_{p, r}(\phi)=\left[L_{p}(N), \Delta_{p, q}(\phi)\right]_{\theta} .
$$

b) We may construct a bounded linear map

$$
\mathcal{Q}_{r}: L_{r}(N \oplus N) \rightarrow \Delta_{p, r}(\phi)
$$

such that

$$
\mathcal{Q}_{r}\left(d^{\frac{1}{r}-\frac{1}{p}} x, x d^{\frac{1}{r}-\frac{1}{p}}\right)=x \text { for all } x \in L_{p}(N) .
$$

In particular, $j_{p, r} \mathcal{Q}_{r}$ is a projection from $L_{r}(N \oplus N)$ onto $j_{p, r}\left(\Delta_{p, r}(\phi)\right)$.

The relevant constants can be estimated as functions of $p, q, r$ in both cases.

We refer the reader to the end of this section for a more general form of Theorem 1.1 .

Remark 1.2. The isomorphism in a) satisfies

$$
\left(\left\|d^{\frac{1}{r}-\frac{1}{p}} x\right\|_{r}^{r}+\left\|x d^{\frac{1}{r}-\frac{1}{p}}\right\|_{r}^{r}\right)^{\frac{1}{r}} \sim\|x\|_{\left[L_{p}(N), \Delta_{p, q}(\phi)\right]_{\theta}} \quad \text { for all } \quad x \in L_{p}(N) .
$$

Remark 1.3. As we shall justify below, the adjoint mapping $\mathcal{Q}_{r}^{*}: \Delta_{p, r}(\phi)^{*} \rightarrow L_{r^{\prime}}(N \oplus N)$ has the form $\mathcal{Q}_{r}^{*}(\xi)=(u(\xi), u(\xi))$ for some bounded linear map $u: \Delta_{p, r}(\phi)^{*} \rightarrow L_{r^{\prime}}(N)$. Equivalently, we have $\mathcal{Q}_{r}(y,-y)=0$ for all $y$.

It is not very convenient to prove the result for an arbitrary density $d$. We will apply a well-known construction of Haagerup and reduce the problem to the case where $N$ is a finite von Neumann algebra and $d, d^{-1}$ are bounded. Moreover, by elementary functional calculus, we may then assume that

$$
d=\sum_{k=1}^{n} d_{k} e_{k}
$$

where the $e_{k}$ are disjoint projections with $\sum_{k} e_{k}=1$ and $d_{k}$ are strictly positive numbers such that $d_{1} \leq d_{2} \leq \cdots \leq d_{n}$, see below for justifying this simplification. Therefore, we will assume in what follows (unless stated otherwise) that $N$ is finite and that $d$ satisfies (1.1). We note nevertheless that Theorem 1.1 is formulated for Haagerup $L_{p}$ spaces and hence valid for arbitrary states. For the moment, we work with a finite von Neumann 
algebra and thus we can work with the usual definition of noncommutative $L_{p}$ spaces. In particular, all $L_{p}$ spaces are contained in the space of $\tau$-measurable operators, see [Nel74] for further definitions.

In order to sketch our strategy for the proof of part a) in Theorem 1.1, we need to introduce a more convenient terminology which will be instrumental in the sequel. Let $d$ be a density in $L_{1}(N)$ satisfying (1.1) and let us write $L_{0}(N)$ for the space of $\tau$-measurable operators affiliated to $N$. Then, given $\alpha \in \mathbb{R}$ and $1 \leq q \leq \infty$, we define the spaces

$$
\begin{aligned}
& L_{q}^{r}\left(N, d^{\alpha}\right)=\left\{x \in L_{0}(N) \mid d^{\alpha} x \in L_{q}(N)\right\}, \\
& L_{q}^{c}\left(N, d^{\alpha}\right)=\left\{x \in L_{0}(N) \mid x d^{\alpha} \in L_{q}(N)\right\},
\end{aligned}
$$

equipped with the following norms

$$
\|x\|_{L_{q}^{r}\left(N, d^{\alpha}\right)}=\left\|d^{\alpha} x\right\|_{q} \quad \text { and } \quad\|x\|_{L_{q}^{c}\left(N, d^{\alpha}\right)}=\left\|x d^{\alpha}\right\|_{q} .
$$

Then, we consider the intersection spaces

$$
\Delta_{q}\left(N, d^{\alpha}\right)=L_{q}^{r}\left(N, d^{\alpha}\right) \cap L_{q}^{c}\left(N, d^{\alpha}\right)
$$

so that we can recover $\Delta_{p, q}(\phi)$ with $d$ as in (1.1) as follows

$$
\Delta_{p, q}(\phi)=\Delta_{q}\left(N, d^{\frac{1}{q}-\frac{1}{p}}\right) .
$$

The isomorphism in Theorem 1.1 a) is equivalent to

$$
\Delta_{q_{\theta}}\left(N, d^{\alpha_{\theta}}\right)=\left[\Delta_{q_{0}}\left(N, d^{\alpha_{0}}\right), \Delta_{q_{1}}\left(N, d^{\alpha_{1}}\right)\right]_{\theta}
$$

where $\left(q_{0}, q_{1}, q_{\theta}\right)=(p, q, r)$ and $\left(\alpha_{0}, \alpha_{1}, \alpha_{\theta}\right)=\left(0,1 / q-1 / p, \theta \alpha_{1}\right)$. As usual we may and will understand intersections as the diagonal subspaces of the corresponding direct sum spaces. That is, we have

$$
\Delta_{q_{j}}\left(N, d^{\alpha_{j}}\right) \subset L_{q_{j}}^{r}\left(N, d^{\alpha_{j}}\right) \oplus L_{q_{j}}^{c}\left(N, d^{\alpha_{j}}\right), \quad \text { for } \quad j=0,1 .
$$

By Kosaki's theorem, the components in the direct sum interpolate isometrically. The easiest way to show that these intersections commute with interpolation is to show that there is one projection acting on both spaces $L_{q_{j}}^{r}\left(N, d^{\alpha_{j}}\right) \oplus L_{q_{j}}^{c}\left(N, d^{\alpha_{j}}\right)$ for $j=0,1$ which projects onto the intersection $\Delta_{q_{j}}\left(N, d^{\alpha_{j}}\right)$. The projection will not be constructed on $L_{q_{j}}^{r}\left(N, d^{\alpha_{j}}\right) \oplus L_{q_{j}}^{c}\left(N, d^{\alpha_{j}}\right)$ but on spaces of upper and lower triangular elements.

The core of our argument relies on Schur multipliers. This will be made possible by the canonical embedding $\pi: N \rightarrow M_{n}(N)$ given by

$$
\pi(x)=\sum_{i, j=1}^{n} e_{i j} \otimes e_{i} x e_{j} .
$$


Let us write $\tau$ to denote the trace functional on $N$. This will allow us to distinguish it from the standard trace $\operatorname{tr}$ on $M_{n}$. Note that $\pi$ is a normal (not unital) $*$-homomorphism and we have $(\operatorname{tr} \otimes \tau) \circ \pi=\tau$. Moreover, the mapping $\mathrm{E}: M_{n}(N) \rightarrow \pi(N)$ given by $\mathrm{E}(z)=\pi(1) z \pi(1)$ defines a normal conditional expectation. For the $L_{p}$-version of the map $\pi$, we first introduce the normal faithful state $\psi(x)=\sum_{k} d_{k} \tau\left(x_{k k}\right)$ on $M_{n}(N)$ with associated density

$$
\delta=\left(\sum_{k=1}^{n} d_{k} e_{k k}\right) \otimes 1
$$

With this choice, the map $\pi_{p}: L_{p}(N) \rightarrow L_{p}\left(M_{n}(N)\right)$

$$
\pi_{p}\left(d^{\frac{1-\eta}{p}} x d^{\frac{\eta}{p}}\right)=\delta^{\frac{1-\eta}{p}} \pi(x) \delta^{\frac{\eta}{p}}
$$

becomes an isometric embedding and $\mathrm{E}: L_{p}\left(M_{n}(N)\right) \rightarrow \pi_{p}\left(L_{p}(N)\right)$ still defines a positive contraction, see JX03] for further details. Note that $\pi_{p}^{*}$ takes $\left(x_{i j}\right) \in L_{p^{\prime}}\left(M_{n}(N)\right)$ to $\sum_{i j} e_{i} x_{i j} e_{j} \in L_{p^{\prime}}(N)$, so that $\pi_{p^{\prime}}^{*} \pi_{p}=i d_{L_{p}(N)}$ and $\pi_{p} \pi_{p^{\prime}}^{*}=\mathrm{E}$. Our main tool are the spaces of lower and upper triangular matrices in $M_{n}(N)$ defined as follows

$$
\begin{aligned}
U T_{p} & =\left\{\left(x_{i j}\right) \in L_{p}\left(M_{n}(N)\right) \mid x_{i j}=0 \text { for } i>j\right\}, \\
L T_{p} & =\left\{\left(x_{i j}\right) \in L_{p}\left(M_{n}(N)\right) \mid x_{i j}=0 \text { for } i \leq j\right\} .
\end{aligned}
$$

We shall use the fact that $U T_{p}$ and $L T_{p}$ are interpolation scales. This result was proved by Pisier in [Pis92, Pis93] and provides a noncommutative analogue of the Peter Jones theorem on interpolation of Hardy spaces. We will use the version given in [PX03].

Theorem 1.4 (Pisier/Xu). If $1 \leq p, q \leq \infty$ and $\frac{1}{r}=\frac{1-\theta}{p}+\frac{\theta}{q}$

$$
U T_{r}=\left[U T_{p}, U T_{q}\right]_{\theta} \quad \text { and } \quad L T_{r}=\left[L T_{p}, L T_{q}\right]_{\theta}
$$

hold with equivalent norms. The constants are uniformly bounded in $n$.

Let us note that for $1<q, p<\infty$ this result follows immediately from the well-known fact that $U T_{p}$ and $L T_{p}$ are complemented subspaces of $L_{p}\left(M_{n}(N)\right)$. Indeed, the triangular projection $\mathbf{T}\left(x_{i j} \otimes e_{i j}\right)=\delta_{i \leq j}\left(x_{i j} \otimes e_{i j}\right)$ defines a bounded operator on $L_{p}\left(M_{n}(N)\right)$ with norm controlled by $c \max \left\{p, p^{\prime}\right\}$. Using $\mathbf{T}$ and $1-\mathbf{T}$ for $p$ and $q$, the interpolation result follows immediately. The whole point of Pisier's argument is to extend this result to the non-trivial borderline cases $q=1$ and $p=\infty$.

In our result we are interested in subspaces of $L_{p}(N)$ which have upper or lower diagonal form. Moreover, we have to take different powers of the density $d$ into account. This leads to consider the following four norms

$$
\|x\|_{U T_{q}^{r}\left(N, d^{\alpha}\right)}=\left\|\sum_{i \leq j} d_{i}^{\alpha} e_{i} x e_{j}\right\|_{q}, \quad \quad\|x\|_{U T_{q}^{c}\left(N, d^{\alpha}\right)}=\left\|\sum_{i \leq j} e_{i} x e_{j} d_{j}^{\alpha}\right\|_{q},
$$




$$
\|x\|_{L T_{q}^{r}\left(N, d^{\alpha}\right)}=\left\|\sum_{i>j} d_{i}^{\alpha} e_{i} x e_{j}\right\|_{q}, \quad \quad\|x\|_{L T_{q}^{c}\left(N, d^{\alpha}\right)}=\left\|\sum_{i>j} e_{i} x e_{j} d_{j}^{\alpha}\right\|_{q} .
$$

Then we define the associated spaces

$$
\begin{aligned}
U T_{q}^{r}\left(N, d^{\alpha}\right) & =\left\{x \in L_{0}(N) \mid e_{i} x e_{j}=0 \text { for } i>j,\|x\|_{U T_{q}^{r}\left(N, d^{\alpha}\right)}<\infty\right\}, \\
L T_{q}^{r}\left(N, d^{\alpha}\right) & =\left\{x \in L_{0}(N) \mid e_{i} x e_{j}=0 \text { for } i \leq j,\|x\|_{L T_{q}^{r}\left(N, d^{\alpha}\right)}<\infty\right\}, \\
U T_{q}^{c}\left(N, d^{\alpha}\right) & =\left\{x \in L_{0}(N) \mid e_{i} x e_{j}=0 \text { for } i>j,\|x\|_{U T_{q}^{c}\left(N, d^{\alpha}\right)}<\infty\right\}, \\
L T_{q}^{c}\left(N, d^{\alpha}\right) & =\left\{x \in L_{0}(N) \mid e_{i} x e_{j}=0 \text { for } i \leq j,\|x\|_{L T_{q}^{c}\left(N, d^{\alpha}\right)}<\infty\right\} .
\end{aligned}
$$

We shall also need to use the spaces

$$
\begin{aligned}
U T_{q}^{r}\left(M_{n}(N), \delta^{\alpha}\right) & =\left\{\left(x_{i j}\right) \in L_{0}\left(M_{n}(N)\right) \mid x_{i j}=0 \text { for } i>j,\left\|\delta^{\alpha}\left(x_{i j}\right)\right\|_{q}<\infty\right\} \\
L T_{q}^{r}\left(M_{n}(N), \delta^{\alpha}\right) & =\left\{\left(x_{i j}\right) \in L_{0}\left(M_{n}(N)\right) \mid x_{i j}=0 \text { for } i \leq j,\left\|\delta^{\alpha}\left(x_{i j}\right)\right\|_{q}<\infty\right\}, \\
U T_{q}^{c}\left(M_{n}(N), \delta^{\alpha}\right) & =\left\{\left(x_{i j}\right) \in L_{0}\left(M_{n}(N)\right) \mid x_{i j}=0 \text { for } i>j,\left\|\left(x_{i j}\right) \delta^{\alpha}\right\|_{q}<\infty\right\} \\
L T_{q}^{c}\left(M_{n}(N), \delta^{\alpha}\right) & =\left\{\left(x_{i j}\right) \in L_{0}\left(M_{n}(N)\right) \mid x_{i j}=0 \text { for } i \leq j,\left\|\left(x_{i j}\right) \delta^{\alpha}\right\|_{q}<\infty\right\} .
\end{aligned}
$$

Let us observe that, if $e_{i} x e_{j}=0$ for $i>j$, we have for $\alpha=\frac{1}{q}-\frac{1}{p}$

$$
\pi_{q}\left(\sum_{i \leq j} d_{i}^{\alpha} e_{i} x e_{j}\right)=\pi_{q}\left(d^{\alpha} x\right)=\delta^{\alpha} \pi_{p}(x) .
$$

In particular, it is easily seen that

$$
\mathrm{E}: U T_{q}^{r}\left(M_{n}(N), \delta^{\alpha}\right) \rightarrow \pi_{p}\left(U T_{q}^{r}\left(N, d^{\alpha}\right)\right)
$$

is still a contractive projection. This property (which extends automatically to the three other spaces considered above) will be instrumental in the following result, where we combine Kosaki's embedding with interpolation of triangular matrices.

Lemma 1.5. If $1 \leq q_{0}, q_{1} \leq \infty$ and $\alpha_{0}, \alpha_{1} \in \mathbb{R}$, let us take $1 / q_{\theta}=(1-\theta) / q_{0}+\theta / q_{1}$ and $\alpha_{\theta}=(1-\theta) \alpha_{0}+\theta \alpha_{1}$. Then, the following isomorphisms hold with relevant constants depending only on $q_{0}, q_{1}$ and $\theta$

$$
\begin{aligned}
& {\left[U T_{q_{0}}^{r}\left(N, d^{\alpha_{0}}\right), U T_{q_{1}}^{r}\left(N, d^{\alpha_{1}}\right)\right]_{\theta}=U T_{q_{\theta}}^{r}\left(N, d^{\alpha_{\theta}}\right),} \\
& {\left[L T_{q_{0}}^{r}\left(N, d^{\alpha_{0}}\right), L T_{q_{1}}^{r}\left(N, d^{\alpha_{1}}\right)\right]_{\theta}=L T_{q_{\theta}}^{r}\left(N, d^{\alpha_{\theta}}\right),} \\
& {\left[U T_{q_{0}}^{c}\left(N, d^{\alpha_{0}}\right), U T_{q_{1}}^{c}\left(N, d^{\alpha_{1}}\right)\right]_{\theta}=U T_{q_{\theta}}^{c}\left(N, d^{\alpha_{\theta}}\right),} \\
& {\left[L T_{q_{0}}^{c}\left(N, d^{\alpha_{0}}\right), L T_{q_{1}}^{c}\left(N, d^{\alpha_{1}}\right)\right]_{\theta}=L T_{q_{\theta}}^{c}\left(N, d^{\alpha_{\theta}}\right) .}
\end{aligned}
$$


Proof. Since the proof of the four isomorphisms is identical, we only consider the first one. According to the boundedness of (1.4), it suffices to prove the analogous isomorphism on the amplified algebra $M_{n}(N)$

$$
\left[U T_{q_{0}}^{r}\left(M_{n}(N), \delta^{\alpha_{0}}\right), U T_{q_{1}}^{r}\left(M_{n}(N), \delta^{\alpha_{1}}\right)\right]_{\theta}=U T_{q_{\theta}}^{r}\left(M_{n}(N), \delta^{\alpha_{\theta}}\right) .
$$

Indeed, Kosaki's interpolation theorem tells us that

$$
\left[L_{q_{0}}^{r}\left(M_{n}(N), \delta^{\alpha_{0}}\right), L_{q_{1}}^{r}\left(M_{n}(N), \delta^{\alpha_{1}}\right)\right]_{\theta}=L_{q_{\theta}}^{r}\left(M_{n}(N), \delta^{\alpha_{\theta}}\right)
$$

holds isometrically. Thus, by our special choice of $\delta$, we obtain a contractive inclusion

$$
\left[U T_{q_{0}}^{r}\left(M_{n}(N), \delta^{\alpha_{0}}\right), U T_{q_{1}}^{r}\left(M_{n}(N), \delta^{\alpha_{1}}\right)\right]_{\theta} \subset U T_{q_{\theta}}^{r}\left(M_{n}(N), \delta^{\alpha_{\theta}}\right) .
$$

For the converse, we assume that $x \in U T_{q_{\theta}}^{r}\left(M_{n}(N), \delta^{\alpha_{\theta}}\right)$ has norm less than 1 . That is, $x \in L_{0}\left(M_{n}(N)\right)$ is an upper triangular matrix such that $\left\|\delta^{\alpha_{\theta}} x\right\|_{q_{\theta}}<1$. Let $\mathcal{S}$ stand for the strip $\mathcal{S}=\{z \in \mathbb{C} \mid 0 \leq \operatorname{Re}(z) \leq 1\}$ and denote by $\left(\partial_{0}, \partial_{1}\right)$ the left and right sides of its boundary. According to Theorem 1.4, we may find an analytic function

$$
f: \mathcal{S} \rightarrow U T_{q_{0}}+U T_{q_{1}}
$$

such that $f(\theta)=\delta^{\alpha_{\theta}} x$ and

$$
\max \left\{\sup _{z \in \partial_{0}}\|f(z)\|_{U T_{q_{0}}}, \sup _{z \in \partial_{1}}\|f(z)\|_{U T_{q_{1}}}\right\} \leq c\left(q_{\theta}\right)
$$

holds for some universal constant $c\left(q_{\theta}\right)$. Then we define $g(z)=\delta^{-(1-z) \alpha_{0}-z \alpha_{1}} f(z)$. Note that $g$ is analytic and that $g(z)$ is still an upper triangular matrix for any $z \in \mathcal{S}$. For $z \in \partial_{0}$ we find

$$
\|g(z)\|_{U T_{q_{0}}^{r}\left(M_{n}(N), \delta^{\alpha} 0\right)}=\|f(z)\|_{U T_{q_{0}}} \leq c\left(q_{\theta}\right) .
$$

Similarly, if $z \in \partial_{1}$ we have the estimate

$$
\|g(z)\|_{U T_{q_{1}}^{r}\left(M_{n}(N), \delta^{\left.\alpha_{1}\right)}\right.}=\|f(z)\|_{U T_{q_{1}}} \leq c\left(q_{\theta}\right) .
$$

Clearly we have $g(\theta)=x$ and (1.5) follows from the three lines lemma.

The next lemma is a very well-known classical result. We have decided to include the proof for the convenience of the reader. The easy argument that we use here is due to Burak Erdogan.

Lemma 1.6. Let $f: \mathbb{R} \rightarrow \mathbb{R}$ be an even integrable function whose restriction to $\mathbb{R}_{+}$is non-increasing and convex. Assume that $f$ is differentiable almost everywhere and $f^{\prime}$ is integrable. Then $f$ is positive definite, i.e. its Fourier transform is positive. 
Proof. If $\xi \in \mathbb{R}_{+}$, we have

$$
\begin{aligned}
\widehat{f}(\xi) & =\int_{\mathbb{R}} f(x) e^{-i x \xi} d x=2 \int_{\mathbb{R}_{+}} f(x) \cos (x \xi) d x \\
& =-\frac{2}{\xi} \int_{\mathbb{R}_{+}} f^{\prime}(x) \sin (x \xi) d x=-\frac{2}{\xi^{2}} \int_{\mathbb{R}_{+}} f^{\prime}\left(\frac{x}{\xi}\right) \sin (x) d x
\end{aligned}
$$

Here we used the fact that $f$ is even, integration by parts and substitution. The function $g(x)=-f^{\prime}\left(\frac{x}{\xi}\right)$ is positive, non-increasing and integrable on $\mathbb{R}_{+}$. In particular, we deduce that

$$
\gamma_{k}=\int_{0}^{2 \pi} g(x+2 \pi k) \sin (x) d x \geq 0
$$

for all integer $k \geq 0$ and therefore

$$
\widehat{f}(\xi)=\frac{2}{\xi^{2}} \sum_{k \geq 0} \gamma_{k} \geq 0 \text { for all } x \in \mathbb{R}_{+} .
$$

By symmetry, $\widehat{f}(\xi) \geq 0$ for all $\xi \neq 0$. Moreover, since $f$ is positive, we have

$$
\widehat{f}(0)=\int_{\mathbb{R}} f(x) d x \geq 0 .
$$

This shows that $\widehat{f}: \mathbb{R} \rightarrow \mathbb{R}_{+}$, so that $f$ is positive definite and the proof is complete.

Lemma 1.7. Let $a=\left(\sum_{k} a_{k} e_{k k}\right) \otimes 1$ be a positive density on $M_{n}(N)$ with non-decreasing entries $a_{1} \leq a_{2} \leq \cdots \leq a_{n}$. Let $\mathcal{L}_{a}(x)=$ ax and $\mathcal{R}_{a}(x)=x a$ be the left and right multiplication maps. Then, the norm of the maps

$$
\mathcal{L}_{a^{\eta}} \mathcal{R}_{a^{1-\eta}}\left(\mathcal{L}_{a}+\mathcal{R}_{a}\right)^{-1} \quad(0 \leq \eta \leq 1)
$$

on the spaces $U T_{p}$ and $L T_{p}$ is bounded by $\frac{3}{2}$ for all $1 \leq p \leq \infty$. In particular, given $\alpha, \beta \in \mathbb{R}$ and $d$ a density as in (1.1), the norm of the following maps is also bounded by $\frac{3}{2}$ on $U T_{q}^{r / c}\left(N, d^{\alpha}\right)$ and $L T_{q}^{r / c}\left(N, d^{\alpha}\right)$ for all $1 \leq q \leq \infty$ and all $0 \leq \eta \leq 1$

$$
\mathcal{L}_{d^{(1-\eta) \beta}} \mathcal{R}_{d^{\eta \beta}}\left(\mathcal{L}_{d^{\beta}}+\mathcal{R}_{d^{\beta}}\right)^{-1}
$$

Proof. Let $x \in U T_{p}$ be an upper triangular matrix. Then we observe that

$$
\mathcal{L}_{a}\left(\mathcal{L}_{a}+\mathcal{R}_{a}\right)^{-1}\left(x_{i j}\right)=\left(\frac{a_{i}}{a_{i}+a_{j}} x_{i j}\right)=\left(\frac{\min \left(a_{i}, a_{j}\right)}{a_{i}+a_{j}} x_{i j}\right)
$$

because for $i>j$ we have $x_{i j}=0$. Observe that the same argument shows that on $L T_{p}$ we have to use $\max \left(a_{i}, a_{j}\right)$ instead of $\min \left(a_{i}, a_{j}\right)$. However, we have $\frac{\max \left(a_{i}, a_{j}\right)}{a_{i}+a_{j}}=1-\frac{\min \left(a_{i}, a_{j}\right)}{a_{i}+a_{j}}$. 
Therefore, the cases $\eta=0,1$ follow immediately once we have shown that

$$
M_{a}\left(x_{i j}\right)=\left(\frac{\min \left(a_{i}, a_{j}\right)}{a_{i}+a_{j}} x_{i j}\right)
$$

is bounded on $L_{p}\left(M_{n}(N)\right)$ for all $1 \leq p \leq \infty$. If $s, t \in \mathbb{R}_{+}$, we have

$$
\frac{\min (s, t)}{s+t}=\frac{1}{1+\frac{\max (s, t)}{\min (s, t)}}=\frac{1}{1+e^{|\log (s)-\log (t)|}} .
$$

The Fourier inversion formula for $f(x)=\frac{1}{1+e^{|x|}}$ gives

$$
\frac{\min (s, t)}{s+t}=\frac{1}{1+e^{|\log (s)-\log (t)|}}=\frac{1}{2 \pi} \int_{\mathbb{R}} \widehat{f}(\xi) e^{i \xi(\log (s)-\log (t))} d \xi .
$$

According to Lemma 1.6, $f$ is positive definite and we obtain

$$
\begin{aligned}
\left\|M_{a}\left(x_{i j}\right)\right\|_{p} & =\left\|\left(\frac{1}{2 \pi} \int_{\mathbb{R}} \widehat{f}(\xi) e^{i \xi\left(\log \left(a_{i}\right)-\log \left(a_{j}\right)\right)} x_{i j} d \xi\right)\right\|_{p} \\
& \leq \frac{1}{2 \pi} \int_{\mathbb{R}} \widehat{f}(\xi)\left\|\left(e^{i \xi \log \left(a_{i}\right)} x_{i j} e^{-i \xi \log \left(a_{j}\right)}\right)\right\|_{p} d \xi \\
& \leq \frac{1}{2 \pi} \int_{\mathbb{R}} \widehat{f}(\xi) d \xi\left\|\left(x_{i j}\right)\right\|_{p}=\frac{\left\|\left(x_{i j}\right)\right\|_{p}}{1+e^{0}}=\frac{1}{2}\left\|\left(x_{i j}\right)\right\|_{p} .
\end{aligned}
$$

Thus, $M_{a}$ is bounded on $L_{p}\left(M_{n}(N)\right)$ with norm $\frac{1}{2}$ and the same holds for $\mathcal{L}_{a}\left(\mathcal{L}_{a}+\mathcal{R}_{a}\right)^{-1}$ on the space $U T_{p}$. Moreover, the same arguments show that $\mathcal{R}_{a}\left(\mathcal{L}_{a}+\mathcal{R}_{a}\right)^{-1}$ is bounded on $L T_{p}$ with norm $\frac{1}{2}$. On the other hand, recalling one more time that $\frac{\max \left(a_{i}, a_{j}\right)}{a_{i}+a_{j}}+\frac{\min \left(a_{i}, a_{j}\right)}{a_{i}+a_{j}}=1$, we deduce that $\mathcal{L}_{a}\left(\mathcal{L}_{a}+\mathcal{R}_{a}\right)^{-1}$ on $L T_{p}$ and $\mathcal{R}_{a}\left(\mathcal{L}_{a}+\mathcal{R}_{a}\right)^{-1}$ on $U T_{p}$ are respectively bounded by $1+\frac{1}{2}$. It remains to prove the case $0<\eta<1$. Let us consider $x \in U T_{p}$ and define the complex function $f(z)=\mathcal{L}_{a^{1-z}} \mathcal{R}_{a^{z}}\left(\mathcal{L}_{a}+\mathcal{R}_{a}\right)^{-1}(x)$. Then it is easily seen that

$$
\max \left\{\sup _{z \in \partial_{0}}\|f(z)\|_{p}, \sup _{z \in \partial_{1}}\|f(z)\|_{p}\right\} \leq \frac{3}{2}\|x\|_{p} .
$$

Thus, we find that $\|f(\eta)\|_{p} \leq \frac{3}{2}\|x\|_{p}$. The argument for $L T_{p}$ is similar. Let us now prove the second assertion. Since the left and right multiplication maps $\mathcal{L}$ and $\mathcal{R}$ clearly commute with $d^{\alpha}$, it is no restriction to assume that $\alpha=0$ and $q=p$. On the other hand, taking

$$
z_{i j}=d_{i}^{(1-\eta) \beta} \frac{e_{i} x e_{j}}{d_{i}^{\beta}+d_{j}^{\beta}} d_{j}^{\eta \beta}
$$

we clearly have

$$
\mathcal{L}_{\delta^{(1-\eta) \beta}} \mathcal{R}_{\delta^{\eta \beta}}\left(\mathcal{L}_{\delta^{\beta}}+\mathcal{R}_{\delta^{\beta}}\right)^{-1} \pi_{p}(x)=\sum_{i j} e_{i j} \otimes z_{i j}=\pi_{p} \mathcal{L}_{d^{(1-\eta) \beta}} \mathcal{R}_{d^{\eta \beta}}\left(\mathcal{L}_{d^{\beta}}+\mathcal{R}_{d^{\beta}}\right)^{-1}(x) .
$$

Therefore, the first assertion implies the second assertion and we are done. 
In the following we use the notations

$$
\begin{aligned}
U T_{q}(N) & =U T_{q}^{r}\left(N, d^{0}\right)=U T_{q}^{c}\left(N, d^{0}\right), \\
L T_{q}(N) & =L T_{q}^{r}\left(N, d^{0}\right)=L T_{q}^{c}\left(N, d^{0}\right), \\
\Delta_{q}^{U T}\left(N, d^{\alpha}\right) & =U T_{q}^{r}\left(N, d^{\alpha}\right) \cap U T_{q}^{c}\left(N, d^{\alpha}\right), \\
\Delta_{q}^{L T}\left(N, d^{\alpha}\right) & =L T_{q}^{r}\left(N, d^{\alpha}\right) \cap L T_{q}^{c}\left(N, d^{\alpha}\right),
\end{aligned}
$$

for spaces of upper and lower triangular elements.

Lemma 1.8. Let $1 \leq q_{0}, q_{1} \leq \infty, \alpha \in \mathbb{R}$ and $\alpha_{\theta}=\theta \alpha$. Then the map

$$
\Lambda: U T_{q_{\theta}}^{r}\left(N, d^{\alpha_{\theta}}\right) \oplus U T_{q_{\theta}}^{c}\left(N, d^{\alpha_{\theta}}\right) \rightarrow\left[U T_{q_{0}}(N), \Delta_{q_{1}}^{U T}\left(N, d^{\alpha}\right)\right]_{\theta}
$$

defined by

$$
\Lambda(y, z)=\left(\mathcal{L}_{d^{\alpha} \theta}+\mathcal{R}_{d^{\alpha} \theta}\right)^{-1}\left(d^{\alpha_{\theta}} y+z d^{\alpha_{\theta}}\right)
$$

satisfies $\|\Lambda\| \leq c\left(q_{\theta}\right)$. The same holds for the space of lower triangular matrices.

Proof. According to Lemma 1.5, we know that

$$
\begin{aligned}
U T_{q_{\theta}}^{r}\left(N, d^{\alpha_{\theta}}\right) & =\left[U T_{q_{0}}(N), U T_{q_{1}}^{r}\left(N, d^{\alpha}\right)\right]_{\theta}, \\
U T_{q_{\theta}}^{c}\left(N, d^{\alpha_{\theta}}\right) & =\left[U T_{q_{0}}(N), U T_{q_{1}}^{c}\left(N, d^{\alpha}\right)\right]_{\theta},
\end{aligned}
$$

holds up to a constant $c^{\prime}\left(q_{\theta}\right)$. Obviously, we have $\Lambda(x, x)=x$. Therefore, it suffices to show that $\Lambda$ is bounded on $U T_{q_{0}}(N) \oplus U T_{q_{0}}(N)$ and on $U T_{q_{1}}^{r}\left(N, d^{\alpha}\right) \oplus U T_{q_{1}}^{c}\left(N, d^{\alpha}\right)$. Indeed, we deduce from Lemma 1.7 that

$$
\begin{aligned}
\|\Lambda(y, z)\|_{U T_{q_{0}}(N)} & =\left\|\mathcal{L}_{d^{\alpha}}\left(\mathcal{L}_{d^{\alpha}}+\mathcal{R}_{d^{\alpha}}\right)^{-1}(y)+\mathcal{R}_{d^{\alpha}}\left(\mathcal{L}_{d^{\alpha}}+\mathcal{R}_{d^{\alpha}}\right)^{-1}(z)\right\|_{q_{0}} \\
& \leq \frac{3}{2}\|y\|_{q_{0}}+\frac{3}{2}\|z\|_{q_{0}} \leq 3\|(y, z)\|_{U T_{q_{0}}(N) \oplus U T_{q_{0}}(N)} .
\end{aligned}
$$

On the other hand, we have

$$
\begin{aligned}
\|\Lambda(y, z)\|_{U T_{q_{1}}^{r}\left(N, d^{\alpha}\right)} & =\left\|\mathcal{L}_{d^{\alpha}}\left(\mathcal{L}_{d^{\alpha}}+\mathcal{R}_{d^{\alpha}}\right)^{-1}\left(d^{\alpha} y+z d^{\alpha}\right)\right\|_{q_{1}} \leq \frac{3}{2}\left\|d^{\alpha} y+z d^{\alpha}\right\|_{q_{1}} \\
& \leq 3 \max \left\{\left\|d^{\alpha} y\right\|_{q_{1}},\left\|z d^{\alpha}\right\|_{q_{1}}\right\}=3\|(y, z)\|_{U T_{q_{1}}^{r}\left(N, d^{\alpha}\right) \oplus U T_{q_{1}}^{c}\left(N, d^{\alpha}\right)} .
\end{aligned}
$$

The estimate for $U T_{q_{1}}^{c}\left(N, d^{\alpha}\right)$ uses $\mathcal{R}_{d^{\alpha}}\left(\mathcal{L}_{d^{\alpha}}+\mathcal{R}_{d^{\alpha}}\right)^{-1}$ instead. On the other hand, the proof for lower triangular matrices is verbatim the same. The proof is complete.

The next result is well-known. It can be proved using the fact that $L_{p}(N)$ are UMD spaces (see [BGM86] and [Bou86]) or applying the boundedness for the noncommutative Hilbert transform in chapter 8 of [PX03], see also the earlier results in [GK69, KP70]. 
Lemma 1.9. Let $\left(e_{i}\right)$ be a family of disjoint projections in a von Neumann algebra $N$ and let us consider the triangular projection $\mathbf{T}_{e}(x)=\sum_{i \leq j} e_{i} x e_{j}$. Then, the mapping $\mathbf{T}_{e}$ is bounded on $L_{p}(N)$ for $1<p<\infty$.

Proof. It is well-known that the triangular projection $\mathbf{T}(a)=\sum_{i \leq j} a_{i j} e_{i j}$ is completely bounded on $S_{p}$, see the references above. Then, the bounded map $\pi_{p^{\prime}}^{*} \mathbf{T} \pi_{p}$ yields the modified triangular projection $\mathbf{T}_{e}$ used in the assertion.

Step 1 of the proof. We will prove Theorem 1.1 assuming (1.1). For the first assertion a), we observe from Kosaki's interpolation that the inclusion $\left[L_{p}(N), \Delta_{p, q}(\phi)\right]_{\theta} \subset \Delta_{p, r}(\phi)$ is trivially contractive. For the converse we use the $\Delta_{q}\left(N, d^{\alpha}\right)$ terminology. In other words we have to prove that

$$
\Delta_{r}\left(N, d^{\theta / s}\right) \subset\left[L_{p}(N), \Delta_{q}\left(N, d^{1 / s}\right)\right]_{\theta} \quad \text { with } \quad \frac{1}{s}=\frac{1}{q}-\frac{1}{p} .
$$

On the other hand, the inclusions $U T_{p}(N) \subset L_{p}(N)$ and $\Delta_{q}^{U T}\left(N, d^{1 / s}\right) \subset \Delta_{q}\left(N, d^{1 / s}\right)$ are contractive and the same happens for the spaces of lower triangular matrices. Therefore, considering the decomposition $x=\mathbf{T}_{e}(x)+x-\mathbf{T}_{e}(x)$ for $x \in \Delta_{r}\left(N, d^{\theta / s}\right)$, it suffices to show that

$$
\begin{aligned}
& \Delta_{r}^{U T}\left(N, d^{\theta / s}\right) \subset\left[U T_{p}(N), \Delta_{q}^{U T}\left(N, d^{1 / s}\right)\right]_{\theta}, \\
& \Delta_{r}^{L T}\left(N, d^{\theta / s}\right) \subset\left[L T_{p}(N), \Delta_{q}^{L T}\left(N, d^{1 / s}\right)\right]_{\theta} .
\end{aligned}
$$

Note that $1<r<\infty$ because $0<\theta<1$. According to Lemma 1.9, this implies that $\mathbf{T}_{e}(x)$ belongs to $\Delta_{r}^{U T}\left(N, d^{\theta / s}\right)$ and $x-\mathbf{T}_{e}(x) \in \Delta_{r}^{L T}\left(N, d^{\theta / s}\right)$. Hence, applying Lemma 1.8 we deduce that

$$
\begin{aligned}
\left\|\mathbf{T}_{e}(x)\right\|_{\left[U T_{p}(N), \Delta_{q}^{U T}\left(N, d^{1 / s}\right)\right]_{\theta}} & =\left\|\Lambda\left(\mathbf{T}_{e}(x), \mathbf{T}_{e}(x)\right)\right\|_{\left[U T_{p}(N), \Delta_{q}^{U T}\left(N, d^{1 / s}\right)\right]_{\theta}} \\
& \leq c(r)\left\|\mathbf{T}_{e}(x)\right\|_{\Delta_{r}^{U T}\left(N, d^{\theta / s}\right)} \leq c(r) d(r)\|x\|_{\Delta_{r}\left(N, d^{\theta / s}\right)} .
\end{aligned}
$$

The same argument with respect to lower triangular matrices gives

$$
\left\|x-\mathbf{T}_{e}(x)\right\|_{\left[L T_{p}(N), \Delta_{q}^{L T}\left(N, d^{1 / s}\right)\right]_{\theta}} \leq c(r) d(r)\|x\|_{\Delta_{r}\left(N, d^{\theta / s}\right)} .
$$

For the proof of part b) we construct

$$
\mathcal{Q}_{r}: L_{r}(N \oplus N) \rightarrow \Delta_{p, r}(\phi)
$$

as follows

$$
\mathcal{Q}_{r}(y, z)=\left(\mathcal{L}_{d^{\frac{1}{r}-\frac{1}{p}}}+\mathcal{R}_{d^{\frac{1}{r}-\frac{1}{p}}}\right)^{-1}(y+z)
$$


for $y, z \in L_{r}(N)$. Clearly, we have $\mathcal{Q}_{r}\left(d^{\frac{1}{r}-\frac{1}{p}} x, x d^{\frac{1}{r}-\frac{1}{p}}\right)=x$ for all $x \in L_{p}(N)$. For the norm estimate, we use again the fact that the triangular map $\mathbf{T}_{e}$ is bounded. For $y, z \in L_{r}(N)$ we deduce from Lemma 1.7 that

$$
\begin{aligned}
\left\|\mathcal{Q}_{r}\left(\mathbf{T}_{e}(y), \mathbf{T}_{e}(z)\right)\right\|_{U T_{r}^{r}\left(N, d^{\frac{1}{r}-\frac{1}{p}}\right)} & =\left\|\mathcal{L}_{d^{\frac{1}{r}-\frac{1}{p}}}\left(\mathcal{L}_{d^{\frac{1}{r}-\frac{1}{p}}}+\mathcal{R}_{d^{\frac{1}{r}-\frac{1}{p}}}\right)^{-1}\left(\mathbf{T}_{e}(y)+\mathbf{T}_{e}(z)\right)\right\|_{r} \\
& \leq 3\left\|\left(\mathbf{T}_{e}(y), \mathbf{T}_{e}(z)\right)\right\|_{L_{r}(N \oplus N)} \leq 3 d(r)\|(y, z)\|_{L_{r}(N \oplus N)}
\end{aligned}
$$

where $d(r)$ stands for the norm of the triangular projection on $L_{r}(N)$. The same estimate holds for $U T_{r}^{c}\left(N, d^{\frac{1}{r}-\frac{1}{p}}\right)$. We can also repeat the estimate for $y-\mathbf{T}_{e}(y)$ and $z-\mathbf{T}_{e}(z)$ with respect to the spaces $L T_{r}^{r}\left(N, d^{\frac{1}{r}-\frac{1}{p}}\right)$ and $L T_{r}^{c}\left(N, d^{\frac{1}{r}-\frac{1}{p}}\right)$. This yields the norm estimate

$$
\left\|\mathcal{Q}_{r}: L_{r}(N \oplus N) \rightarrow \Delta_{p, r}(\phi)\right\| \leq 6 d(r)
$$

Remark 1.10. In our applications we will combine a) and b) and deduce that

$$
\left\|\mathcal{Q}_{r}: L_{r}(N \oplus N) \rightarrow\left[L_{p}(N), \Delta_{p, q}(\phi)\right]_{\theta}\right\| \leq 6 c(r) d(r)^{2} .
$$

Here we use the triangular projection twice. In the first version of this paper we directly constructed a map $\widehat{\mathcal{Q}}_{r}: L_{r}(N \oplus N) \rightarrow\left[L_{p}(N), \Delta_{p, q}(\phi)\right]_{\theta}$ projecting onto the canonical image of $\Delta_{p, r}(\phi)$. Indeed, we consider upper triangular elements $y=d^{\frac{1}{r}-\frac{1}{p}} x_{1}, z=x_{2} d^{\frac{1}{r}-\frac{1}{p}}$ with $x_{1}, x_{2} \in U T_{p}(N)$. Then the "canonical" image in $L_{q}(N \oplus N)$ is given by

$$
\left(d^{\frac{1}{q}-\frac{1}{r}} y, z d^{\frac{1}{q}-\frac{1}{r}}\right)=\left(d^{\frac{1}{q}-\frac{1}{p}} x_{1}, x_{2} d^{\frac{1}{q}-\frac{1}{p}}\right) .
$$

We have seen in Lemma 1.8 that

$$
\left\|\Lambda\left(x_{1}, x_{2}\right)\right\|_{\Delta_{q}^{U T}\left(N, d^{\alpha}\right)} \leq 3\left\|\left(d^{\frac{1}{q}-\frac{1}{r}} y, z d^{\frac{1}{q}-\frac{1}{r}}\right)\right\|_{L_{q}(N \oplus N)} \quad \text { for } \quad \alpha=1 / q-1 / p .
$$

The same estimate holds with respect to $L T_{p}(N)$. By complex interpolation we deduce

$$
\begin{aligned}
& \left\|\Lambda\left(x_{1}, x_{2}\right)\right\|_{\left[U T_{p}(N), \Delta_{q}^{U T}\left(N, d^{\frac{1}{q}-\frac{1}{p}}\right)\right]_{\theta}} \\
& \quad \leq 3\left\|\left(x_{1}, x_{2}\right)\right\|_{\left[U T_{p}(N) \oplus U T_{p}(N), U T_{q}^{r}\left(N, d^{\frac{1}{q}-\frac{1}{p}}\right) \oplus U T_{q}^{c}\left(N, d^{\frac{1}{q}-\frac{1}{p}}\right)\right]_{\theta}} \\
& \quad \leq 3 c(r)\left\|\left(x_{1}, x_{2}\right)\right\|_{U T_{r}^{r}\left(N, d^{\frac{1}{r}-\frac{1}{p}}\right) \oplus U T_{r}^{c}\left(N, d^{\frac{1}{r}-\frac{1}{p}}\right)}=3 c(r)\|(y, z)\|_{L_{r}(N \oplus N) .} .
\end{aligned}
$$

For $y, z \in L_{r}(N)$ we consider $\zeta=d^{\frac{1}{q}-\frac{1}{r}} y+z d^{\frac{1}{q}-\frac{1}{r}}$ and the projection

$$
\widehat{\mathcal{Q}}_{r}(y, z)=\left(d^{\frac{1}{q}-\frac{1}{p}}\left(\left(\mathcal{L}_{d^{\frac{1}{q}-\frac{1}{p}}}+\mathcal{R}_{d^{\frac{1}{q}-\frac{1}{p}}}\right)^{-1}(\zeta)\right),\left(\left(\mathcal{L}_{d^{\frac{1}{q}-\frac{1}{p}}}+\mathcal{R}_{d^{\frac{1}{q}-\frac{1}{p}}}\right)^{-1}(\zeta)\right) d^{\frac{1}{q}-\frac{1}{p}}\right) .
$$

Then we have

$$
\left\|\widehat{\mathcal{Q}}_{r}: L_{r}(N \oplus N) \rightarrow\left[L_{p}(N), \Delta_{p, q}(\phi)\right]_{\theta}\right\| \leq 6 c(r) d(r) .
$$

It is known that $d(r) \leq c \max \left\{r, r^{\prime}\right\}$. However, we have no explicit control on $c(r)$. It would be interesting to know whether the singularity for $r \rightarrow 1$ is necessary when interpolating $\left[N, \Delta_{\infty, 1}(\phi)\right]_{\frac{1}{r}}$. 
Remark 1.11. In contrast to $\widehat{\mathcal{Q}}_{r}$, the projection from part b) satisfies the condition $\mathcal{Q}_{r}(y,-y)=0$ mentioned in Remark 1.3. This follows immediately from (1.6) and is important for our applications below. Let us reformulate this condition for the dual map. Using $\mathcal{Q}_{r}(y,-y)=0$ we see that $\mathcal{Q}_{r}$ factors through

$$
L_{r}(N) \simeq L_{r}(N \oplus N) /\left\{(y,-y) \mid y \in L_{r}(N)\right\} .
$$

More explicitly, for $\xi \in \Delta_{p, r}(\phi)^{*}$ we have

$$
\begin{aligned}
\left\langle\mathcal{Q}_{r}^{*}(\xi),(y, z)\right\rangle & =\left\langle\xi, \mathcal{Q}_{r}(y, z)\right\rangle=\left\langle\xi,\left(\mathcal{L}_{d^{\frac{1}{r}-\frac{1}{p}}}+\mathcal{R}_{d^{\frac{1}{r}-\frac{1}{p}}}\right)^{-1}(y+z)\right\rangle \\
& =\left\langle\xi,\left(\mathcal{L}_{d^{\frac{1}{r}-\frac{1}{p}}}+\mathcal{R}_{d^{\frac{1}{r}-\frac{1}{p}}}\right)^{-1}(y)\right\rangle+\left\langle\xi,\left(\mathcal{L}_{d^{\frac{1}{r}-\frac{1}{p}}}+\mathcal{R}_{d^{\frac{1}{r}-\frac{1}{p}}}\right)^{-1}(z)\right\rangle .
\end{aligned}
$$

This allows us to define the bounded map $u(\xi)$ by $\langle u(\xi), y\rangle=\frac{1}{2}\left\langle\mathcal{Q}_{r}^{*}(\xi),(y, y)\right\rangle$. Clearly, we have $\mathcal{Q}_{r}^{*}(\xi)=(u(\xi), u(\xi))$. Assuming (1.1) the map $\left(\mathcal{L}_{d^{\frac{1}{r}-\frac{1}{p}}}+\mathcal{R}_{d^{\frac{1}{r}-\frac{1}{p}}}\right)^{-1}$ is bounded. In the next steps of our proof this is not necessarily the case, but see Corollary 1.16 below.

Step 2 of the proof. We now study the case where $N$ is finite and equipped with a density $d$ such that $c_{1} 1 \leq d \leq c_{2} 1$ for some constants $0<c_{1} \leq c_{2}<\infty$, so that $d$ and $d^{-1}$ are bounded. We claim that for any $\varepsilon>0$ we may find a density $d_{\varepsilon}$ of the form (1.1), with $\tau\left(d_{\varepsilon}\right)=1$ and such that

$$
(1+\varepsilon)^{-1} d_{\varepsilon} \leq d \leq(1+\varepsilon) d_{\varepsilon} .
$$

Indeed, let $\mu$ be the probability measure on the Borel $\sigma$-algebra over $\left[c_{1}, c_{2}\right]$ determined by $\mu(\mathrm{E})=\tau\left(1_{\mathrm{E}}(d)\right)$, where $1_{\mathrm{E}}(d)$ denotes the corresponding spectral projection. This provides isometric isomorphisms $L_{p}(\mu)=L_{p}(A, \tau)$, where $A$ is the (abelian) von Neumann subalgebra of $N$ given by

$$
A=\left\{f(d) \mid f:\left[c_{1}, c_{2}\right] \rightarrow \mathbb{C} \text { bounded and measurable }\right\} .
$$

In particular, we may approximate $d$ by $d_{\varepsilon}$ of the form (1.1) just by approximating the function $f(x)=x$ by a suitable simple function. In particular, we may even assume that $d_{\varepsilon}$ commutes with $d$. Letting $\phi_{\varepsilon}(x)=\operatorname{tr}\left(d_{\varepsilon} x\right)$ be the state determined by $d_{\varepsilon}$ and taking $\frac{1}{s}=\frac{1}{q}-\frac{1}{p}$, it is clear that

$$
(1+\varepsilon)^{\frac{-1}{s}}\|x\|_{\Delta_{p, q}\left(\phi_{\varepsilon}\right)} \leq\|x\|_{\Delta_{p, q}(\phi)} \leq(1+\varepsilon)^{\frac{1}{s}}\|x\|_{\Delta_{p, q}\left(\phi_{\varepsilon}\right)} .
$$

This gives an $(1+\varepsilon)^{\frac{2}{s}}$-isomorphism

$$
\left[L_{p}(N), \Delta_{p, q}(\phi)\right]_{\theta}=\left[L_{p}(N), \Delta_{p, q}\left(\phi_{\varepsilon}\right)\right]_{\theta} .
$$

In addition, $\Delta_{p, r}(\phi)=\Delta_{p, r}\left(\phi_{\varepsilon}\right)$ are $(1+\varepsilon)^{\frac{2}{u}}$-isomorphic with $\frac{1}{u}=\frac{1}{r}-\frac{1}{p}$ and

$$
\Delta_{p, r}(\phi)=\Delta_{p, r}\left(\phi_{\varepsilon}\right)=\left[L_{p}(N), \Delta_{p, q}\left(\phi_{\varepsilon}\right)\right]_{\theta}=\left[L_{p}(N), \Delta_{p, q}(\phi)\right]_{\theta} .
$$


This proves the first assertion. Let us denote by $I_{\varepsilon}: \Delta_{p, r}\left(\phi_{\varepsilon}\right) \rightarrow \Delta_{p, r}(\phi)$ the formal identity. Let $\mathcal{Q}_{r}(\varepsilon): L_{r}(N \oplus N) \rightarrow \Delta_{p, r}\left(\phi_{\varepsilon}\right)$ be the projection constructed above. Then we denote by $\mathcal{Q}_{r}: L_{r}(N \oplus N) \rightarrow \Delta_{p, r}(\phi)$ the densely defined map

$$
\mathcal{Q}_{r}\left(d^{\frac{1}{u}} \alpha, \beta d^{\frac{1}{u}}\right)=I_{\varepsilon} \mathcal{Q}_{r}(\varepsilon)\left(d_{\varepsilon}^{\frac{1}{u}} \alpha, \beta d_{\varepsilon}^{\frac{1}{u}}\right)
$$

Since we have $\left\|\mathcal{Q}_{r}\right\| \leq(1+\varepsilon)^{\frac{1}{r}-\frac{1}{p}}\left\|\mathcal{Q}_{r}(\varepsilon)\right\|$, it turns out that $\mathcal{Q}_{r}$ is the desired projection.

Remark 1.12. Let us explain how we may pass to the limit $\varepsilon \rightarrow 0$ for the definition of $\mathcal{Q}_{r}$. We denote by $B_{\infty}(\mathbb{R})$ the algebra of bounded measurable functions on $\mathbb{R}$ and find a normal *-representation $\pi: B_{\infty}(\mathbb{R}) \otimes_{\min } B_{\infty}(\mathbb{R}) \rightarrow B\left(L_{2}(N)\right)$ given by $\pi(f \otimes g)=\mathcal{L}_{f(d)} \mathcal{R}_{g(d)}$. This shows that

$$
\mathcal{L}_{d^{1 / u}}\left(\mathcal{L}_{d^{1 / u}}+\mathcal{R}_{d^{1 / u}}\right)^{-1}=\mathrm{SOT}-\lim _{\varepsilon \rightarrow 0} \mathcal{L}_{d_{\varepsilon}^{1 / u}}\left(\mathcal{L}_{d_{\varepsilon}^{1 / u}}+\mathcal{R}_{d_{\varepsilon}^{1 / u}}\right)^{-1}
$$

A similar statement holds for $\mathcal{R}_{d^{1 / u}}\left(\mathcal{L}_{d^{1 / u}}+\mathcal{R}_{d^{1 / u}}\right)^{-1}$. Therefore, for $x \in L_{r}(N)$ the family $T_{\varepsilon}(x)=\mathcal{L}_{d_{\varepsilon}^{1 / u}}\left(\mathcal{L}_{d_{\varepsilon}^{1 / u}}+\mathcal{R}_{d_{\varepsilon}^{1 / u}}\right)^{-1}(x)$ is uniformly bounded in $L_{r}(N)$ and converges in $L_{2}(N)$. It follows very easily from [FK86, Theorem 3.6] that $T_{\varepsilon}(x)$ converges in $L_{r}(N)$. We recall the canonical embedding $j_{p, r}: \Delta_{p, r}(\phi) \rightarrow L_{r}(N \oplus N)$ given by $j_{p, r}(x)=\left(\mathcal{L}_{d^{1 / u}} x, \mathcal{R}_{d^{1 / u}} x\right)$ and deduce that

$$
j_{p, r}\left(\mathcal{L}_{d^{1 / u}}+\mathcal{R}_{d^{1 / u}}\right)^{-1}: L_{r}(N \oplus N) \rightarrow L_{r}(N \oplus N)
$$

is a well-defined bounded map. Thus $\mathcal{Q}_{r}=\left(\mathcal{L}_{d^{1 / u}}+\mathcal{R}_{d^{1 / u}}\right)^{-1}$ is a projection onto $\Delta_{p, r}(\phi)$ and the pointwise limit of the $\mathcal{Q}_{r}(\varepsilon)^{\prime}$ 's. In particular, the condition from Remark 1.3 is satisfied. Indeed, using the Borel functional calculus for $B_{\infty}(\mathbb{R}) \otimes B_{\infty}(\mathbb{R})$ we find

$$
\mathcal{Q}_{r}(y, z)=\int_{\mathbb{R} \times \mathbb{R}}\left(d(\omega)^{1 / u}+d\left(\omega^{\prime}\right)^{1 / u}\right)^{-1} d E_{\omega}(y+z) d E_{\omega^{\prime}} .
$$

Let us note that in the semifinite case (without assuming $c_{1} \leq d \leq c_{2}$ but still assuming $d$ is faithful), we may obtain the same formula by using an increasing net of spectral projections of $d$.

The proof for the general case is based on Haagerup's reduction theorem, see [JXb]. Let us briefly explain how this construction works. Let us consider a von Neumann algebra $N$ equipped with a normal faithful state $\phi$ associated to a density $d$. Let us define the discrete group

$$
\mathrm{G}=\bigcup_{n \in \mathbb{N}} 2^{-n} \mathbb{Z}
$$

Then we construct the crossed product $M=N \rtimes_{\sigma^{\phi}}$ G. That is, if $H$ is the Hilbert space provided by the GNS construction applied to $\phi$ and $\sigma^{\phi}$ denotes the one parameter modular 
automorphism group on $N$ associated to $\phi$, then $M$ is generated by the representations $\pi: N \rightarrow \mathcal{B}\left(L_{2}(\mathrm{G} ; H)\right)$ and $\lambda: \mathrm{G} \rightarrow \mathcal{B}\left(L_{2}(\mathrm{G} ; H)\right)$, where

$$
(\pi(x) \xi)(g)=\sigma_{-g}^{\phi}(x) \xi(g) \quad \text { and } \quad(\lambda(h) \xi)(g)=\xi(g-h) .
$$

By the faithfulness of $\pi$ we are allowed to identify $N$ with its image $\pi(N)$. Then, a generic element in the crossed product $M$ has the form $\sum_{g} x_{g} \lambda(g)$ with $x_{g} \in N$ and we have the conditional expectation

$$
\mathrm{E}_{N}\left(\sum_{g \in \mathrm{G}} x_{g} \lambda(g)\right)=x_{0} \in N
$$

The algebra $M$ contains an increasing net $\left(M_{\alpha}\right)_{\alpha \in \Lambda}$ of finite von Neumann subalgebras with normal conditional expectations $\mathcal{E}_{\alpha}: M \rightarrow M_{\alpha}$. One of the important properties of Haagerup's construction is that $\psi=\phi \circ \mathrm{E}_{N}$ is a normal faithful state such that $\psi \circ \mathcal{E}_{\alpha}=\psi$ holds for each $\alpha \in \Lambda$. Moreover, the restriction $\psi_{\alpha}$ of $\psi$ to $M_{\alpha}$ has a density $d_{\alpha}$ such that

$$
c_{1}(\alpha) 1_{M_{\alpha}} \leq d_{\alpha} \leq c_{2}(\alpha) 1_{M_{\alpha}}
$$

for some constants $0<c_{1}(\alpha) \leq c_{2}(\alpha)<\infty$. If $d_{\psi}$ denotes the density associated to the state $\psi$, we consider the canonical conditional expectation $\mathcal{E}_{\alpha, p}: L_{p}(M) \rightarrow L_{p}\left(M_{\alpha}\right)$ and the canonical inclusion $\iota_{\alpha, p}: L_{p}\left(M_{\alpha}\right) \rightarrow L_{p}(M)$ densely defined respectively by

$$
\mathcal{E}_{\alpha, p}\left(x d_{\psi}^{\frac{1}{p}}\right)=\mathcal{E}_{\alpha}(x) d_{\alpha}^{\frac{1}{p}} \quad \text { and } \quad \iota_{\alpha, p}\left(x d_{\alpha}^{\frac{1}{p}}\right)=x d_{\psi}^{\frac{1}{p}} .
$$

We refer to [JX03] for more information on these maps. It is shown in [JXb] that

$$
\lim _{\alpha} \iota_{\alpha, p} \mathcal{E}_{\alpha, p}(x)=x \text { for all } \quad x \in L_{p}(M) \text { and } 1 \leq p<\infty .
$$

We will also need the $L_{p}$ version of $\mathrm{E}_{N}: L_{p}(M) \rightarrow L_{p}(N)$ :

$$
\mathrm{E}_{N, p}\left(x d_{\psi}^{\frac{1}{p}}\right)=\mathrm{E}_{N}(x) d^{\frac{1}{p}}
$$

This comes with the natural inclusion map $j_{N, p}: L_{p}(N) \rightarrow L_{p}(M), j_{N, p}\left(x d^{\frac{1}{p}}\right)=x d_{\psi}^{\frac{1}{p}}$, see again [JX03]. With this information we start our approximation procedure. Indeed, the following mappings will be instrumental in our proof of Theorem 1.1 for general von Neumann algebras

$$
u_{\alpha, p}=\mathrm{E}_{N, p} \iota_{\alpha, p}: L_{p}\left(M_{\alpha}\right) \rightarrow L_{p}(N) \quad \text { and } \quad w_{\alpha, p}=\mathcal{E}_{\alpha, p} j_{N, p}: L_{p}(N) \rightarrow L_{p}\left(M_{\alpha}\right) .
$$

Lemma 1.13. The following properties hold:

i) If $1 \leq p<\infty, \lim _{\alpha} u_{\alpha, p} w_{\alpha, p}(x)=x$ for all $x \in L_{p}(N)$.

ii) The mappings $u_{\alpha, p}$ and $w_{\alpha, p}$ induce contractions

$$
\begin{array}{cl}
u_{\alpha, p}: & {\left[L_{p}\left(M_{\alpha}\right), \Delta_{p, q}\left(\psi_{\alpha}\right)\right]_{\theta} \rightarrow\left[L_{p}(N), \Delta_{p, q}(\phi)\right]_{\theta},} \\
w_{\alpha, p}: & {\left[L_{p}(N), \Delta_{p, q}(\phi)\right]_{\theta} \rightarrow\left[L_{p}\left(M_{\alpha}\right), \Delta_{p, q}\left(\psi_{\alpha}\right)\right]_{\theta} .}
\end{array}
$$


iii) If $1 \leq q<p \leq \infty$ and $0<\theta<1$, we have

$$
\lim _{\alpha} u_{\alpha, p} w_{\alpha, p}(x)=x \quad \text { for all } \quad x \in\left[L_{p}(N), \Delta_{p, q}(\phi)\right]_{\theta} .
$$

Proof. Since $\mathrm{E}_{N, p} j_{N, p}(x)=x$ for all $x \in L_{p}(N)$, we have

$$
\lim _{\alpha} u_{\alpha, p} w_{\alpha, p}(x)-x=\lim _{\alpha} \mathrm{E}_{N, p}\left(\iota_{\alpha, p} \mathcal{E}_{\alpha, p}\left(j_{N, p}(x)\right)-j_{N, p}(x)\right)=0,
$$

where the last identity follows from (1.8) and the contractivity of $\mathrm{E}_{N, p}$ in $L_{p}(M)$. This proves the first assertion. Now let us identify $\Delta_{p, q}$ with its image $j_{p, q}\left(\Delta_{p, q}\right)$ in $L_{q}(N \oplus N)$ and also $\Delta_{p, q}\left(\psi_{\alpha}\right)$ with its image $j_{p, q}\left(\Delta_{p, q}\left(\psi_{\alpha}\right)\right)$ in $L_{q}\left(M_{\alpha} \oplus M_{\alpha}\right)$. Then, to prove ii) we will regard the mapping

$$
w_{\alpha, p}: \Delta_{p, q}(\phi) \rightarrow \Delta_{p, q}\left(\psi_{\alpha}\right)
$$

as the restriction of $w_{\alpha, q} \oplus w_{\alpha, q}: L_{q}(N \oplus N) \rightarrow L_{q}\left(M_{\alpha} \oplus M_{\alpha}\right)$ to the subspace

$$
\left\{\left(d^{\frac{1}{s}} x, x d^{\frac{1}{s}}\right) \mid x \in L_{p}(N)\right\} \quad \text { with } \quad 1 / s=1 / q-1 / p .
$$

If $x=y d^{\frac{1}{p}}$ for $y \in N$, we have

$$
\begin{aligned}
w_{\alpha, q}\left(j_{p, q}(x)\right) & =\left(w_{\alpha, q}\left(d^{\frac{1}{s}} x\right), w_{\alpha, q}\left(x d^{\frac{1}{s}}\right)\right) \\
& =\left(d_{\alpha}^{\frac{1}{s}} \mathcal{E}_{\alpha}(y) d_{\alpha}^{\frac{1}{p}}, \mathcal{E}_{\alpha}(y) d_{\alpha}^{\frac{1}{q}}\right)=j_{p, q}\left(\mathcal{E}_{\alpha}(y) d_{\alpha}^{\frac{1}{p}}\right)=j_{p, q}\left(w_{\alpha, p}(x)\right) .
\end{aligned}
$$

Here we use the well-known fact that $\mathcal{E}_{\alpha, p}\left(d_{\psi}^{\frac{1-\eta}{p}} x d_{\psi}^{\frac{\eta}{p}}\right)=d_{\alpha}^{\frac{1-\eta}{p}} \mathcal{E}_{\alpha}(x) d_{\alpha}^{\frac{\eta}{p}}$, which follows from our definition of $\mathcal{E}_{\alpha, p}$ and the identity $\mathcal{E}_{\alpha} \sigma^{\psi}=\sigma^{\psi_{\alpha}} \mathcal{E}_{\alpha}$, see [JX03] for further details. Therefore the map $w_{\alpha, p}$ induces a compatible contraction on the interpolation couple $\left(L_{p}(N), \Delta_{p, q}(\phi)\right)$ and hence on the complex interpolation space $\left[L_{p}(N), \Delta_{p, q}(\phi)\right]_{\theta}$. The argument for $u_{\alpha, p}$ is entirely similar. In the proof of iii) we first observe that it suffices to prove the assertion on a dense subspace, because we already know from ii) that the maps $u_{\alpha, p} w_{\alpha, p}$ are contractions. If $x \in L_{p}(N)$ (we remind the reader that $p=\infty$ is allowed and hence we may not assume that $\lim _{\alpha} u_{\alpha, p} w_{\alpha, p}(x)=x$ holds in norm), we set $\gamma_{\alpha, p}=u_{\alpha, p} w_{\alpha, p}$ and have

$$
\begin{aligned}
& \lim _{\alpha}\left\|\gamma_{\alpha, q}(x)-x\right\|_{\left[L_{p}(N), \Delta_{p, q}(\phi)\right]_{\theta}} \\
& \quad \leq \lim _{\alpha}\left\|\gamma_{\alpha, p}(x)-x\right\|_{p}^{1-\theta}\left(\left\|d^{\frac{1}{s}}\left(\gamma_{\alpha, p}(x)-x\right)\right\|_{q}^{q}+\left\|\left(\gamma_{\alpha, p}(x)-x\right) d^{\frac{1}{s}}\right\|_{q}^{q}\right)^{\frac{\theta}{q}} \\
& \leq\left(2\|x\|_{p}\right)^{1-\theta} \lim _{\alpha}\left(\left\|\gamma_{\alpha, q}\left(d^{\frac{1}{s}} x\right)-d^{\frac{1}{s}} x\right\|_{q}^{q}+\left\|\gamma_{\alpha, q}\left(x d^{\frac{1}{s}}\right)-x d^{\frac{1}{s}}\right\|_{q}^{q}\right)^{\frac{\theta}{q}}=0 .
\end{aligned}
$$

The first inequality uses the three lines lemma, the second applies i) and uses $\theta>0$. 
Step 3 of the proof. We now conclude the proof of Theorem 1.1. For the assertion a) we observe that the upper estimate in Remark 1.2 holds in general by the same argument used in Step 1 above. For the lower estimate we observe that $\left(M_{\alpha}, d_{\alpha}\right)$ satisfies the hypotheses of Step 2. Hence we have

$$
\left(\left\|d_{\alpha}^{\frac{1}{r}-\frac{1}{p}} x\right\|_{r}^{r}+\left\|x d_{\alpha}^{\frac{1}{r}-\frac{1}{p}}\right\|_{r}^{r}\right)^{\frac{1}{r}} \sim\|x\|_{\left[L_{p}\left(M_{\alpha}\right), \Delta_{p, q}\left(\psi_{\alpha}\right)\right]_{\theta}}
$$

for all $x \in L_{p}\left(M_{\alpha}\right)$ and $\alpha \in \Lambda$. This implies that

$$
\begin{aligned}
\|x\|_{\left[L_{p}(N), \Delta_{p, q}(\phi)\right]_{\theta}} & \leq \lim \sup _{\alpha}\left\|\gamma_{\alpha, p}(x)\right\|_{\left[L_{p}(N), \Delta_{p, q}(\phi)\right]_{\theta}} \\
& \leq \lim \sup _{\alpha}\left\|w_{\alpha, p}(x)\right\|_{\left[L_{p}\left(M_{\alpha}\right), \Delta_{p, q}\left(\psi_{\alpha}\right)\right]_{\theta}} \\
& \lesssim \lim \sup _{\alpha}\left(\left\|d_{\alpha}^{\frac{1}{r}-\frac{1}{p}} \mathcal{E}_{\alpha, p}(x)\right\|_{r}^{r}+\left\|\mathcal{E}_{\alpha, p}(x) d_{\alpha}^{\frac{1}{r}-\frac{1}{p}}\right\|_{r}^{r}\right)^{\frac{1}{r}} \\
& \leq\left(\left\|d^{\frac{1}{r}-\frac{1}{p}} x\right\|_{r}^{r}+\left\|x d^{\frac{1}{r}-\frac{1}{p}}\right\|_{r}^{r}\right)^{\frac{1}{r}}
\end{aligned}
$$

We will now construct the projection as a suitable limit. Let

$$
\mathcal{Q}_{\alpha, r}: L_{r}\left(M_{\alpha} \oplus M_{\alpha}\right) \rightarrow \Delta_{p, r}\left(\psi_{\alpha}\right)
$$

be the projection from Step 2 and let $\mathcal{U}$ be a free ultrafilter on $\Lambda$. Then we define

$$
\left\langle\mathcal{Q}_{r}(x, y), \xi\right\rangle=\lim _{\alpha, \mathcal{U}}\left\langle u_{\alpha, p} \mathcal{Q}_{\alpha, r}\left(w_{\alpha, r}(x), w_{\alpha, r}(y)\right), \xi\right\rangle
$$

for every $\xi \in \Delta_{p, r}(\phi)^{*}$. Note that $\Delta_{p, r}(\phi)$ is a reflexive Banach space. Therefore, we deduce that we have $\mathcal{Q}_{r}(x, y) \in \Delta_{p, r}(\phi)$ for all $(x, y) \in L_{r}(N \oplus N)$. Since $\mathcal{Q}_{\alpha, r}$ is a projection, we deduce

$$
u_{\alpha, p} \mathcal{Q}_{\alpha, r}\left(w_{\alpha, r}\left(d^{\frac{1}{r}-\frac{1}{p}} x\right), w_{\alpha, r}\left(x d^{\frac{1}{r}-\frac{1}{p}}\right)\right)=u_{\alpha, p} \mathcal{Q}_{\alpha, r}\left(d_{\alpha}^{\frac{1}{r}-\frac{1}{p}} w_{\alpha, p}(x), w_{\alpha, p}(x) d_{\alpha}^{\frac{1}{r}-\frac{1}{p}}\right)=\gamma_{\alpha, p}(x) .
$$

Thus Lemma $1.13 \mathrm{iii}$ ) and $\left[L_{p}(N), \Delta_{p, q}(\phi)\right]_{\theta}=\Delta_{p, r}(\phi)$ imply

$$
\mathcal{Q}_{r}\left(d^{\frac{1}{r}-\frac{1}{p}} x, x d^{\frac{1}{r}-\frac{1}{p}}\right)=x
$$

for all $x \in L_{p}(N)$. Since $\mathcal{Q}_{r}$ is continuous, we deduce the result by density.

Remark 1.14. Theorem 1.1 also holds in the category of operator spaces. That is, the map $\mathcal{Q}_{r}: L_{r}(N \oplus N) \rightarrow \Delta_{p, r}(\phi)$ is completely bounded. This follows immediately from replacing $d$ by $1 \otimes d$ in $L_{1}\left(M_{m}(N)\right)$. Moreover, in the semifinite setting the assumption $\tau(d)=1$ is not really needed. Therefore, Theorem 1.1 also holds for $\tau$-measurable operators $d$. More generally, this can be extended to strictly semifinite weights. At the time of this writing it is not clear whether there is a result in this direction for arbitrary weights. For two densities $d_{1}$ and $d_{2}$ we can obtain results in this direction by considering $(1,2)$ entries in the space $\Delta_{p, r}\left(\phi_{2}\right)$, where $\phi_{2}$ is associated to the density $d=d_{1} \otimes e_{11}+d_{2} \otimes e_{22}$ on $M_{2}(N)$. We leave the details to the interested reader. 
Using the methods of our paper, the referee found a proof for the following interpolation result which generalizes our Theorem 1.1. We are indebted to the referee for allowing us to reproduce his argument.

Theorem 1.15. Let $1 \leq q_{0}, q_{1} \leq \infty$ and $\alpha_{0}, \alpha_{1} \geq 0$. Define $1 / q_{\theta}=(1-\theta) / q_{0}+\theta / q_{1}$ and $\alpha_{\theta}=(1-\theta) \alpha_{0}+\theta \alpha_{1}$ for $0<\theta<1$. Then, the following isomorphism holds for any density $d$ of a normal faithful state on $N$

$$
\Delta_{q_{\theta}}\left(N, d^{\alpha_{\theta}}\right)=\left[\Delta_{q_{0}}\left(N, d^{\alpha_{0}}\right), \Delta_{q_{1}}\left(N, d^{\alpha_{1}}\right)\right]_{\theta} .
$$

Proof. Here we will prove the result assuming (1.1). The proof in the general case follows by approximation in the semifinite case and an application of Haagerup's decomposition, as in Step 3 above. Using the triangular map, it suffices to prove

$$
\Delta_{q_{\theta}}^{U T}\left(N, d^{\alpha_{\theta}}\right)=\left[\Delta_{q_{0}}^{U T}\left(N, d^{\alpha_{0}}\right), \Delta_{q_{1}}^{U T}\left(N, d^{\alpha_{1}}\right)\right]_{\theta} .
$$

According to Lemma 1.5, the direct sums $U T_{q_{\theta}}^{r}\left(N, d^{\alpha_{\theta}}\right) \oplus U T_{q_{\theta}}^{c}\left(N, d^{\alpha_{\theta}}\right)$ are an interpolation scale. Thus, it suffices to find a common projection which is bounded for $q_{0}$ and $q_{1}$. Let us show that the map

$$
\mathcal{Q}(y, z)=(x, x) \quad \text { where } \quad x=\left(\mathcal{L}_{d^{\alpha_{0}+\alpha_{1}}}+\mathcal{R}_{d^{\alpha_{0}+\alpha_{1}}}\right)^{-1}\left(\mathcal{L}_{d^{\alpha_{0}+\alpha_{1}}}(y)+\mathcal{R}_{d^{\alpha_{0}+\alpha_{1}}}(z)\right)
$$

is bounded in both spaces. Indeed, Lemma 1.7 gives

$$
\begin{aligned}
& \left\|\left(\mathcal{L}_{d^{\alpha_{0}+\alpha_{1}}}+\mathcal{R}_{d^{\alpha_{0}+\alpha_{1}}}\right)^{-1} \mathcal{L}_{d^{\alpha_{0}+\alpha_{1}}}(y)\right\|_{U T_{q_{j}}^{r}\left(N, d^{\alpha_{j}}\right)} \leq \frac{3}{2}\|y\|_{U T_{q_{j}}^{r}\left(N, d^{\alpha_{j}}\right)}, \\
& \left\|\left(\mathcal{L}_{d^{\alpha_{0}+\alpha_{1}}}+\mathcal{R}_{d^{\alpha_{0}+\alpha_{1}}}\right)^{-1} \mathcal{R}_{d^{\alpha_{0}+\alpha_{1}}}(z)\right\|_{U T_{q_{j}}^{c}\left(N, d^{\alpha_{j}}\right)} \leq \frac{3}{2}\|z\|_{U T_{q_{j}}^{c}\left(N, d^{\alpha_{j}}\right)},
\end{aligned}
$$

for $j=0,1$. Hence, it remains to see that

$$
\begin{aligned}
\left\|\left(\mathcal{L}_{d^{\alpha_{0}+\alpha_{1}}}+\mathcal{R}_{d^{\alpha_{0}+\alpha_{1}}}\right)^{-1} \mathcal{L}_{d^{\alpha_{0}+\alpha_{1}}}(y)\right\|_{U T_{q_{j}}^{c}\left(N, d^{\alpha_{j}}\right)} & \leq \frac{3}{2}\|y\|_{U T_{q_{j}}^{r}\left(N, d^{\alpha_{j}}\right)}, \\
\left\|\left(\mathcal{L}_{d^{\alpha_{0}+\alpha_{1}}}+\mathcal{R}_{d^{\alpha_{0}+\alpha_{1}}}\right)^{-1} \mathcal{R}_{d^{\alpha_{0}+\alpha_{1}}}(z)\right\|_{U T_{q_{j}}^{r}\left(N, d^{\alpha_{j}}\right)} & \leq \frac{3}{2}\|z\|_{U T_{q_{j}}^{c}\left(N, d^{\alpha_{j}}\right)} .
\end{aligned}
$$

Since all these cross estimates can be handled similarly, we only estimate the first one in the case $j=0$. Using $\eta=\alpha_{0} /\left(\alpha_{0}+\alpha_{1}\right)$ in conjunction with Lemma 1.7 one more time, we obtain

$$
\begin{aligned}
& \left\|\left(\mathcal{L}_{d^{\alpha_{0}}+\alpha_{1}}+\mathcal{R}_{d^{\alpha_{0}+\alpha_{1}}}\right)^{-1} \mathcal{L}_{d^{\alpha_{0}+\alpha_{1}}}(y)\right\|_{U T_{q_{0}}^{c}\left(N, d^{\alpha_{0}}\right)} \\
& \quad=\left\|\mathcal{L}_{d^{\alpha_{1}}} \mathcal{R}_{d^{\alpha_{0}}}\left(\mathcal{L}_{d^{\alpha_{0}}+\alpha_{1}}+\mathcal{R}_{d^{\alpha_{0}}+\alpha_{1}}\right)^{-1} \mathcal{L}_{d^{\alpha_{0}}}(y)\right\|_{U T_{q_{0}}(N)} \\
& \leq \frac{3}{2}\left\|\mathcal{L}_{d^{\alpha_{0}}}(y)\right\|_{U T_{q_{0}}(N)}=\frac{3}{2}\left\|d^{\alpha_{0}} y\right\|_{U T_{q_{0}}(N)}=\frac{3}{2}\|y\|_{U T_{q_{0}}^{r}\left(N, d^{\alpha} 0\right)} .
\end{aligned}
$$

We apply the same arguments (and same "projection") for lower triangular elements. 
The following byproduct of our arguments might be of independent interest.

Corollary 1.16. Let $1<p<\infty$ and $\alpha>0$. Then the maps

$$
\mathcal{R}_{d^{(1-\eta) \alpha}} \mathcal{L}_{d^{\eta \alpha}}\left(\mathcal{L}_{d^{\alpha}}+\mathcal{R}_{d^{\alpha}}\right)^{-1} \quad(0 \leq \eta \leq 1)
$$

are bounded on $L_{p}(N)$ for any density d of a normal faithful state on $N$.

Proof. For $d=\sum_{k=1}^{n} d_{k} e_{k}$ as in (1.1) this follows immediately from Lemma 1.7 and Lemma 1.9. Then we follow the same procedure as in the proof of Theorem 1.1 by first showing it for finite von Neumman algebras with densities bounded above and below, and then apply the Haagerup construction.

\section{Subspaces of NOnCOMmutative $L_{1}$}

In this section we follow Pisier's approach and prove Theorem A, Let us recall the notions of type and cotype from Banach space theory. Given a probability space $\Omega$, let us consider a sequence $\left(\varepsilon_{k}\right)$ of independent Bernoulli random variables equidistributed in \pm 1 . A linear map $T: X \rightarrow Y$ has type $p$ if there exists $c_{1}>0$ such that the inequality below holds for all finite sequences $\left(x_{k}\right)$ in $X$

$$
\left(\mathbb{E}\left\|\sum_{k} \varepsilon_{k} T\left(x_{k}\right)\right\|_{Y}^{2}\right)^{\frac{1}{2}} \leq c_{1}\left(\sum_{k}\left\|x_{k}\right\|_{X}^{p}\right)^{\frac{1}{p}} .
$$

Then $t_{p}(T)=\inf c_{1}$ satisfying the inequality above. A Banach space has type $p$ if $i d_{X}$ has type $p$. We use the standard notation $t_{p}(X)=t_{p}\left(i d_{X}\right)$. A linear map $T: X \rightarrow Y$ is said to be of cotype $q$ if

$$
\left(\sum_{k}\left\|T\left(x_{k}\right)\right\|_{Y}^{q}\right)^{\frac{1}{q}} \leq c_{2}\left(\mathbb{E}\left\|\sum_{k} \varepsilon_{k} x_{k}\right\|_{X}^{2}\right)^{\frac{1}{2}}
$$

We define $c_{q}(T)=\inf c_{2}$, where the infimum is taken over all $c_{2}$ satisfying the inequality above. Again $c_{q}(X)=c_{q}\left(i d_{X}\right)$ for a Banach space $X$. Given a von Neumann algebra $N$, a linear map $T: L_{p}(N) \rightarrow X$ is called $(q,+)$-summing if there exists a constant $c>0$ such that the inequality below holds for all finite sequence $\left(x_{k}\right)$ of positive elements $x_{k} \in L_{p}(N)$

$$
\left(\sum_{k}\left\|T\left(x_{k}\right)\right\|_{X}^{q}\right)^{\frac{1}{q}} \leq c\left\|\sum_{k} x_{k}\right\|_{p} .
$$

We denote $\pi_{q,+}(T)=\inf c$. Let us recall the well-known fact

$$
\pi_{q,+}\left(T: L_{p}(N) \rightarrow X\right) \leq 2 c_{q}(T) .
$$

Indeed, for positive elements $x_{k}$ the order relation implies that

$$
\left\|\sum_{k} \varepsilon_{k} x_{k}\right\|_{p} \leq\left\|\sum_{k, \varepsilon_{k}=1} x_{k}\right\|_{p}+\left\|\sum_{k, \varepsilon_{k}=-1} x_{k}\right\|_{p} \leq 2\left\|\sum_{k} x_{k}\right\|_{p} .
$$


We shall also need the following well-known fact from interpolation [BL76, section 4.7].

Lemma 2.1. Let $\left(A_{0}, A_{1}\right)$ be an interpolation couple of Banach spaces. Assume that $A_{0}$ is contractively included in $A_{1}$ and let $0<\tilde{\eta}<\eta<1$. Then, there exists some absolute constant $c(\eta, \tilde{\eta})$ depending only on $(\eta, \tilde{\eta})$ such that the norm of the inclusion

$$
\left[A_{0}, A_{1}\right]_{\tilde{\eta}} \subset\left[A_{0}, A_{1}\right]_{\eta, 1} \quad \text { is controlled by } c(\eta, \tilde{\eta}) \text {. }
$$

Without assuming full support for $d$ we keep the notation

$$
\|x\|_{\Delta_{p, q}(\phi)}=\max \left\{\left\|d^{\frac{1}{q}-\frac{1}{p}} x\right\|_{q},\left\|x d^{\frac{1}{q}-\frac{1}{p}}\right\|_{q}\right\} .
$$

If we set $\operatorname{supp} d=e$, the expression above vanishes on $(1-e) L_{p}(N)(1-e)$. Relation (2.3) defines a norm on $e L_{p}(N)+L_{p}(N) e$, a complemented subspaces of the quasi-normed space $\left(L_{p}(N),\|\|_{\Delta_{p, q}}\right)$. We will write $\Delta_{p, q}(\phi)$ for the completion of $e L_{p}(N)+L_{p}(N) e$ with respect to this norm. The spaces $e \Delta_{p, q}(\phi) e, e \Delta_{p, q}(\phi)(1-e)$ and $(1-e) \Delta_{p, q}(\phi) e$ are the complemented subspaces of $\Delta_{p, q}(\phi)$ obtained from the closure of $e L_{p}(N) e, e L_{p}(N)(1-e)$ and $(1-e) L_{p}(N) e$ in $\Delta_{p, q}(\phi)$.

Lemma 2.2. Let $d$ be the density of a normal state $\phi$ and let $e$ be the support projection of $d$, so that $\phi$ is faithful on $e N e$. If $\frac{1}{r}=\frac{1-\theta}{p}+\frac{\theta}{q}$, then $\left[e L_{p}(N)+L_{p}(N) e, \Delta_{p, q}(\phi)\right]_{\theta}$ is isomorphic to the direct sum

$$
e \Delta_{p, r}(\phi) e \oplus e L_{r}(N)(1-e) \oplus(1-e) L_{r}(N) e
$$

The restriction of this isomorphism on $e L_{p}(N)+L_{p}(N)$ e is given by

$$
x \mapsto\left(e x e, d^{\frac{1}{r}-\frac{1}{p}} x(1-e),(1-e) x d^{\frac{1}{r}-\frac{1}{p}}\right) .
$$

Proof. By definition, we have

$$
e \Delta_{p, q}(\phi) e=\Delta_{p, q}\left(\phi_{\left.\right|_{e N e}}\right) .
$$

Hence, we can apply Theorem 1.1 and find that

$$
\left[e L_{p}(N) e, e \Delta_{p, q}(\phi) e\right]_{\theta} \simeq e \Delta_{p, r}(\phi) e .
$$

Now we discuss the off-diagonal parts in

$$
\begin{aligned}
{\left[e L_{p}(N)+L_{p}(N) e, \Delta_{p, q}(\phi)\right]_{\theta} } & \simeq\left[e L_{p}(N) e, e \Delta_{p, q}(\phi) e\right]_{\theta} \\
& \oplus\left[e L_{p}(N)(1-e), e \Delta_{p, q}(\phi)(1-e)\right]_{\theta} \\
& \oplus\left[(1-e) L_{p}(N) e,(1-e) \Delta_{p, q}(\phi) e\right]_{\theta} .
\end{aligned}
$$

However, for $x=e x(1-e)$ we have that

$$
\|\operatorname{ex}(1-e)\|_{\Delta_{p, q}(\phi)}=\max \left\{\left\|d^{\frac{1}{q}-\frac{1}{p}} \operatorname{ex}(1-e)\right\|_{q},\left\|\operatorname{ex}(1-e) d^{\frac{1}{q}-\frac{1}{p}}\right\|_{q}\right\}=\left\|d^{\frac{1}{q}-\frac{1}{p}} x(1-e)\right\|_{q} .
$$


A similar remark applies for $x=(1-e) x e$. Therefore, the interpolation space simplifies considerably in the off-diagonal terms. Applying Kosaki's interpolation theorem we formally obtain

$$
\left[d^{\frac{1}{q}-\frac{1}{p}} e L_{p}(N)(1-e), e L_{q}(N)(1-e)\right]_{\theta}=d^{\frac{1}{q}-\frac{1}{r}} L_{r}(N)(1-e) .
$$

However, $\phi$ does not have full support and we can not apply Kosaki's theorem directly. Let $\psi_{1-e}=\lim _{j} \psi_{j}$ be a strictly semifinite weight on $(1-e) N(1-e)$. Then $\psi=\psi_{1-e}+\phi$ is a strictly semifinite weight on $N$. Let $e_{j} \leq 1-e$ be the support of $\psi_{j}$ (with associated density $d_{j}$ ) and $f_{j}=e_{j}+e$. We may apply Kosaki's interpolation theorem for $\phi_{j}=\psi_{j}+\phi$ and the sum of the commuting densities $d_{j}+d$. Then we obtain

$$
\left[\left(d+d_{j}\right)^{\frac{1}{q}-\frac{1}{p}} L_{p}\left(f_{j} N f_{j}\right), L_{q}\left(f_{j} N f_{j}\right)\right]_{\theta}=\left(d+d_{j}\right)^{\frac{1}{q}-\frac{1}{r}} L_{r}\left(f_{j} N f_{j}\right) .
$$

Since the map $W(y)=e y(1-e)$ is a contraction on the spaces at both sides above, we can replace $N$ by $e N(1-e)$ in the isometric isomorphism since the resulting spaces are contractively complemented. Thus we find

$$
\left[d^{\frac{1}{q}-\frac{1}{p}} L_{p}(N) e_{j}, e L_{q}(N) e_{j}\right]_{\theta}=d^{\frac{1}{q}-\frac{1}{r}} L_{r}(N) e_{j} .
$$

Passing to the limit for $j \rightarrow \infty$ yields (2.6). Note that in (2.4) and (2.6) we used different topological vector spaces for the interpolation couple $\left(A_{0}, A_{1}\right)$. In (2.4), $A_{0}+A_{1}=A_{1}$ is the completion of $e L_{p}(N)(1-e)$ with respect to the norm in $\Delta_{p, q}(\phi)$. On the other hand, in (2.6) we use $e L_{q}(N)(1-e)$ as the underlying vector space. Since $d^{\frac{1}{q}-\frac{1}{p}} L_{p}(N)(1-e)$ is dense in $e L_{q}(N)(1-e)$, we have calculated the interpolation space. Thus (2.4), (2.5) and (2.6) imply that

$$
\|\operatorname{ex}(1-e)\|_{\left[e L_{p}(N)(1-e), e \Delta_{p, q}(\phi)(1-e)\right]_{\theta}}=\left\|d^{\frac{1}{r}-\frac{1}{p}} x(1-e)\right\|_{L_{r}(N)} .
$$

Taking adjoints, we obtain the same conclusion for the space $(1-e) L_{p}(N) e$.

Theorem 2.3. Let $X$ be a Banach space and $T: X \rightarrow L_{1}(N)$ be a linear map such that $T^{*}$ has cotype $q$. Then, there exists a density $d$ on $N$ such that, for all $1<p<q^{\prime}$, we may construct a bounded linear map $u: X \rightarrow L_{p}(N)$ satisfying

$$
T(x)=d^{1-\frac{1}{p}} u(x)+u(x) d^{1-\frac{1}{p}} \quad \text { for all } \quad x \in X .
$$

If moreover $X \subset L_{1}(N)$ is a subspace, $u$ is an isomorphic embedding of $X$ into $L_{p}(N)$.

Proof. We recall from (2.2) that $T^{*}: N \rightarrow X^{*}$ is $(q,+)$-summing. Therefore, we deduce from Pisier's factorization theorem [Pis86b, Theorem 3.2] that there exists a state $\phi$ on $N$ such that

$$
\left\|T^{*}(y)\right\|_{X^{*}} \leq c\|y\|^{1-\frac{2}{q}}\left(\phi\left(y y^{*}\right)+\phi\left(y^{*} y\right)\right)^{\frac{1}{q}} .
$$


We use a standard trick (see [Pis04]) to replace $\phi$ by its normal part. Let $\phi_{n}$ be the normal part of $\phi$. Let $\left(s_{\alpha}\right)$ be a net of contractions in $N$ such that $\lim _{\alpha} s_{\alpha}=1$ in the strong operator and the strong* topology and $\lim _{\alpha} \phi\left(s_{\alpha} y s_{\alpha}\right)=\phi_{n}(y)$. Let $y \in N$ and $x \in X$ of norm 1 such that

$$
\left\|T^{*}(y)\right\|_{X^{*}} \leq(1+\varepsilon)\left|T^{*}(y)(x)\right|=(1+\varepsilon)|\operatorname{tr}(y T(x))| .
$$

We may write $T(x)=a=a_{1} a_{2}$ with $a_{i} \in L_{2}(N)$. Note that

$$
\lim _{\alpha} \operatorname{tr}\left(s_{\alpha} y s_{\alpha} a\right)=\lim _{\alpha} \operatorname{tr}\left(y s_{\alpha} a s_{\alpha}\right)=\operatorname{tr}(y a)
$$

because $\lim _{\alpha} s_{\alpha} a s_{\alpha}=\lim _{\alpha} s_{\alpha} a_{1} a_{2} s_{\alpha}=a_{1} a_{2}=a$. Therefore we find that

$$
\begin{aligned}
\left\|T^{*}(y)\right\|_{X^{*}} & \leq(1+\varepsilon) \lim _{\alpha}\left|\operatorname{tr}\left(s_{\alpha} y s_{\alpha} a\right)\right| \\
& \leq c \lim \sup _{\alpha}\left\|s_{\alpha} y s_{\alpha}\right\|^{1-\frac{2}{q}} \lim _{\alpha}\left(\phi\left(s_{\alpha} y s_{\alpha} y^{*} s_{\alpha}\right)+\phi\left(s_{\alpha} y^{*} s_{\alpha} y s_{\alpha}\right)\right)^{\frac{1}{q}} \\
& \leq c \lim \sup _{\alpha}\left\|s_{\alpha} y s_{\alpha}\right\|^{1-\frac{2}{q}} \lim _{\alpha}\left(\phi\left(s_{\alpha} y y^{*} s_{\alpha}\right)+\phi\left(s_{\alpha} y^{*} y s_{\alpha}\right)\right)^{\frac{1}{q}} \\
& \leq c\|y\|^{1-\frac{2}{q}}\left(\phi_{n}\left(y y^{*}\right)+\phi_{n}\left(y^{*} y\right)\right)^{\frac{1}{q}} .
\end{aligned}
$$

Therefore, we may assume with no loss of generality that the state $\phi$ is normal. This means that it is given by $\phi(y)=\operatorname{tr}(d y)$ for some density $d \in L_{1}(N)$. Let $e$ be the support of $d$, so that $\phi$ is faithful on $e N e$. We then have

$$
\left\|T^{*}(y)\right\|_{X^{*}} \leq c\|y\|_{N}^{1-\frac{2}{q}} \max \left\{\left\|d^{\frac{1}{2}} y\right\|_{2},\left\|y d^{\frac{1}{2}}\right\|_{2}\right\}^{2 / q}=c\|y\|_{N}^{1-\frac{2}{q}}\|y\|_{\Delta_{2}(\phi)}^{2 / q} .
$$

Note that $T^{*}((1-e) y(1-e))=0$. According to a well-known result $(c f$. [BL76, p.49]) we have

$$
\left\|T^{*}(y)\right\|_{X^{*}} \leq c\|y\|_{\left[e N+N e, \Delta_{2}(\phi)\right]_{\frac{2}{q}, 1}} \text { for all } y \in N .
$$

Recall that we write $\Delta_{q}(\phi)$ for $\Delta_{\infty, q}(\phi)$. We consider $(\eta, \tilde{\eta})=\left(2 / q, 2 / p^{\prime}\right)$ and observe that $0<\tilde{\eta}<\eta<1$ since $1<p<q^{\prime}$ and $2<q<\infty$ (if $T^{*}$ has cotype 2 it also has cotype $q$ for all $q>2$ ). According to Lemma 2.1 we deduce that

$$
\left\|T^{*}(y)\right\|_{X^{*}} \leq c c(\eta, \tilde{\eta})\|y\|_{\left[e N+N e, \Delta_{2}(\phi)\right]_{\frac{2}{p^{\prime}}}} \text { for all } \quad y \in N
$$

Therefore, the map $T^{*}$ extends to a bounded map $T_{p^{\prime}}^{*}:\left[e N+N e, \Delta_{2}(\phi)\right]_{2 / p^{\prime}} \rightarrow X^{*}$. Let us note that, in accordance with Lemma 2.2, the intersection in this interpolation space is $e N e+e N(1-e)+(1-e) N e$ while by (2.7) the map $T^{*}$ vanishes on the remaining corner $(1-e) N(1-e)$. Let us recall the projection given by Theorem 1.1

$$
\mathcal{Q}_{p^{\prime}}: L_{p^{\prime}}(e N e \oplus e N e) \rightarrow e \Delta_{p^{\prime}}(\phi) e \simeq\left[e N e, e \Delta_{2}(\phi) e\right]_{\tilde{\eta}} .
$$

Using Lemma 2.2 we may define the map $\widetilde{\mathcal{Q}}_{p^{\prime}}: L_{p^{\prime}}(N \oplus N) \rightarrow \Delta_{p^{\prime}}(\phi)$ by

$$
\widetilde{\mathcal{Q}}_{p^{\prime}}\left(y_{1}, y_{2}\right)=\mathcal{Q}_{p^{\prime}}\left(e y_{1} e, e y_{2} e\right) \oplus e y_{1}(1-e) \oplus(1-e) y_{2} e .
$$


Thus by construction we have

$$
T^{*}(y)=T_{p^{\prime}}^{*} \widetilde{\mathcal{Q}}_{p^{\prime}}\left(d^{\frac{1}{p^{\prime}}} y, y d^{\frac{1}{p^{\prime}}}\right)
$$

for all $y \in N$. Unfortunately, $\widetilde{\mathcal{Q}}_{p^{\prime}}$ does not vanish on vectors of the form $(y,-y)$. Therefore we need a slight modification of $\left(\widetilde{\mathcal{Q}}_{p^{\prime}}\right)^{*}$ which allows us to construct one map $u$ as asserted. For this we define a map $v: \Delta_{p^{\prime}}(\phi)^{*} \rightarrow L_{p}(N)$ as follows. According to Lemma 2.2 we have $\Delta_{p^{\prime}}(\phi)^{*}=\left(e \Delta_{p^{\prime}}(\phi) e\right)^{*} \oplus\left(e L_{p^{\prime}}(N)(1-e)\right)^{*} \oplus\left(1-e L_{p^{\prime}}(N) e^{*}\right.$. Following Remark 1.3 we know that $\mathcal{Q}_{p^{\prime}}^{*}(\xi)=\left(v_{e}(\xi), v_{e}(\xi)\right)$ holds for some bounded linear map

$$
v_{e}:\left(e \Delta_{p^{\prime}}(\phi) e\right)^{*} \rightarrow L_{p}(e N e)
$$

Thus we may define

$$
v(\xi)=v_{e}\left(\xi_{e}\right)+\xi_{e, 1-e}+\xi_{1-e, e} \quad \text { where } \xi \text { has the components } \xi=\left(\xi_{e}, \xi_{e, 1-e}, \xi_{1-e, e}\right) .
$$

Under the usual duality bracket $\langle a, b\rangle=\operatorname{tr}(a b)$, we observe that

$$
\left(e L_{p^{\prime}}(N)(1-e)\right)^{*}=(1-e) L_{p}(N) e \quad \text { and } \quad\left((1-e) L_{p^{\prime}}(N) e\right)^{*}=e L_{p}(N)(1-e) .
$$

Therefore, we may and will assume that $\xi_{e, 1-e}=(1-e) \xi_{e, 1-e} e$ and $\xi_{1-e, e}=e \xi_{1-e, e}(1-e)$ are elements in $L_{p}(N)$. Then we observe that

$$
d^{\frac{1}{p^{\prime}}} v(\xi)+v(\xi) d^{\frac{1}{p^{\prime}}}=d^{\frac{1}{p^{\prime}}} v_{e}\left(\xi_{e}\right)+v_{e}\left(\xi_{e}\right) d^{\frac{1}{p^{\prime}}}+d^{\frac{1}{p^{\gamma}}} \xi_{1-e, e}+\xi_{e, 1-e} d^{\frac{1}{p^{\prime}}}
$$

This implies that, for all $y \in N$, we have

$$
\begin{aligned}
& \left.\widetilde{\mathcal{Q}}_{p^{\prime}}\left(d^{\frac{1}{p^{\prime}}} y, y d^{\frac{1}{p^{\prime}}}\right), \xi\right\rangle \\
& \quad=\left\langle\widetilde{Q}_{p^{\prime}}\left(d^{\frac{1}{p^{\prime}}} e y e, e y e d^{\frac{1}{p^{\prime}}}\right), \xi_{e}\right\rangle+\left\langle d^{\frac{1}{p^{\prime}}} y(1-e), \xi_{e, 1-e}\right\rangle+\left\langle(1-e) y d^{\frac{1}{p^{\prime}}}, \xi_{1-e, e}\right\rangle \\
& \quad=\operatorname{tr}\left(v_{e}\left(\xi_{e}\right)\left(d^{\frac{1}{p^{\prime}}} e y e+e y e d^{\frac{1}{p^{\prime}}}\right)\right)+\operatorname{tr}\left(\xi_{e, 1-e} d^{\frac{1}{p^{\prime}}} y(1-e)\right)+\operatorname{tr}\left(\xi_{1-e, e}(1-e) y d^{\frac{1}{p^{\prime}}}\right) \\
& \quad=\operatorname{tr}\left(\left(d^{\frac{1}{p^{\prime}}} v(\xi)+v(\xi) d^{\frac{1}{p^{\prime}}}\right) y\right) .
\end{aligned}
$$

This will allow us to conclude easily. Indeed, we define $u=v\left(T_{p^{\prime}}^{*}\right)^{*}: X \rightarrow L_{p}(N)$. Then we deduce from (2.10) that

$$
\begin{aligned}
\operatorname{tr}\left(\left(d^{\frac{1}{p^{\prime}}} u(x)+u(x) d^{\frac{1}{p^{\prime}}}\right) y\right) & =\left\langle\widetilde{\mathcal{Q}}_{p^{\prime}}\left(d^{\frac{1}{p^{\prime}}} y, y d^{\frac{1}{p^{\prime}}}\right),\left(T_{p^{\prime}}^{*}\right)^{*}(x)\right\rangle \\
& =\left\langle T_{p^{\prime}}^{*} \widetilde{\mathcal{Q}}_{p^{\prime}}\left(d^{\frac{1}{p^{\prime}}} y, y d^{\frac{1}{p^{\prime}}}\right), x\right\rangle=\left\langle T^{*}(y), x\right\rangle=\operatorname{tr}(y T(x))
\end{aligned}
$$

holds for all $y$. This means $T(x)=d^{\frac{1}{p^{\prime}}} u(x)+u(x) d^{\frac{1}{p^{\prime}}}$. Let us now consider the special case $T=\iota_{X}: X \rightarrow L_{1}(N)$ such that $X^{*}$ has cotype $q$. Then the left inverse for $u$ is given by $v(x)=d^{\frac{1}{p^{\prime}}} x+x d^{\frac{1}{p^{\prime}}}$. Clearly, $v$ is bounded and $u$ becomes an isomorphism. 
Remark 2.4. The proof shows that we can construct the same $u$ because we only care about the restriction of $\mathcal{Q}_{p^{\prime}}$ to elements of the form $\left(d^{1 / p^{\prime}} y, y d^{1 / p^{\prime}}\right)$. If $N$ is $\sigma$-finite and semifinite, we may assume $e=1$ and Remark 1.12 gives

$$
u(x)=\int_{\mathbb{R} \times \mathbb{R}}\left[d(s)^{1 / p^{\prime}}+d(t)^{1 / p^{\prime}}\right]^{-1} d E_{s} T(x) d E_{t} .
$$

Proof of Theorem $\mathbf{A}$. The type index of $X$ is defined as

$$
p_{X}=\inf \{p \mid X \text { has type } p\} \text {. }
$$

According to the Krivine-Maurey-Pisier theorem (see e.g. [MS86] or [Pis86c]) we know that for $p=p_{X}$ the spaces $\ell_{p}(n)$ are uniformly contained in $X$. If $p_{X}=1$, we know from [RX03, Theorem 5.1] that $X$ contains $\ell_{1}$. However, this contradicts the reflexivity of $X$. Hence, $p_{X}$ must be strictly bigger that 1 . Let $p_{0}>1$ such that $X$ has type $p_{0}$. This implies that $X^{*}$ has (finite) cotype $p_{0}^{\prime}$ and therefore Theorem 2.3 applies.

Proof of Corollary C. Let $\left(x_{n}\right)$ be subsymmetric in $N_{*}$ and let

$$
X=\operatorname{span}\left\{x_{n} \mid n \geq 1\right\} .
$$

According to (the proof of) Theorem $\mathrm{A}$, if $X$ does not contain $\ell_{1}$ then $X$ is isomorphic to a subspace of $L_{p}(N)$ for some $1<p<2$. Since we know from [JR] that $L_{p}(N)$ is asymptotically symmetric, we deduce that $\left(x_{n}\right)$ is indeed symmetric.

Remark 2.5. Let $\left(x_{n}\right)$ be a subsymmetric sequence in $L_{1}(N)$. A close inspection of [RX03, Proposition 5.3] shows that $\left(x_{n}\right)$ contains a subsequence equivalent to the unit vector basis of $\ell_{1}$ or $\left(x_{n}\right)$ is 1-equiintegrable (equivalently relatively weakly compact). However, a subsymmetric sequence is equivalent to every subsequence. Thus either $\left(x_{n}\right)$ is equivalent to the $\ell_{1}$ basis (hence symmetric) or 1-equiintegrable. Therefore, the only possibility of a subsymmetric, not symmetric sequence, occurs for 1-equiintegrable sequences where the unit ball of $\operatorname{span}\left\{x_{n}: n \in \mathbb{N}\right\}$ is not 1-equiintegrable, see also [RX03, Theorem 5.1].

\section{NiKISHIN-TYPE RESUltS FOR $p$ FINITE}

In the commutative setting, Nikishin type results can be obtained from a careful analysis of the maximal function. Although maximal functions have been recently introduced in the noncommutative setting [Jun02, JXa, they seem not to be applicable for this type of results. Our approach using duality in the noncommutative setting reduces the problem to norm estimates for positive operators. In this section we prove the differential inequality (1) and Theorem B. Let us start with an elementary observation. The result is known 
due to the work of Araki Ara90] and Kosaki [Kos92]. We give a short proof to keep the paper more self-contained.

Lemma 3.1. Let $1 \leq q \leq \infty$ and $\alpha, \beta$ be positive. Then

$$
\left\|\alpha^{\eta} \beta^{\eta}\right\|_{\frac{q}{\eta}} \leq\|\alpha \beta\|_{q}^{\eta} \quad \text { for all } \quad 0<\eta<1 .
$$

Proof. Let us first show this for $\eta=\frac{1}{2}$. Indeed,

$$
\left\|\alpha^{\frac{1}{2}} \beta^{\frac{1}{2}}\right\|_{2 q}^{2}=\left\|\alpha^{\frac{1}{2}} \beta \alpha^{\frac{1}{2}}\right\|_{q} .
$$

Define $f(z)=\alpha^{1-z} \beta \alpha^{z}$ and fix $\lambda=\|\alpha \beta\|_{q}$. We clearly have

$$
\max \left\{\sup _{z \in \partial_{0}}\|f(z)\|_{q}, \sup _{z \in \partial_{1}}\|f(z)\|_{q}\right\} \leq \lambda .
$$

Therefore, we have $\left\|f\left(\frac{1}{2}\right)\right\|_{q} \leq \lambda$ and deduce the assertion for $\eta=1 / 2$. Now we show the inequality for all $\frac{1}{2}<\eta<1$. Take $c \in L_{\left(\frac{q}{\eta}\right)^{\prime}}(N)$ of norm less than 1 . We may write $\eta / q=(1-\theta) / q+\theta / 2 q$ for some $0<\theta<1$. Now we use interpolation and assume that $N$ is $\sigma$-finite. The general case follows from a well-known approximation argument. Using Kosaki's interpolation theorem, we find an analytic function $g: \mathcal{S} \rightarrow L_{(2 q)^{\prime}}(N)$ such that $g(\theta)=c$ and

$$
\max \left\{\sup _{z \in \partial_{0}}\|g(z)\|_{q^{\prime}}, \sup _{z \in \partial_{1}}\|g(z)\|_{(2 q)^{\prime}}\right\} \leq 1 .
$$

Therefore, the function

$$
h(z)=\operatorname{tr}\left(g(z) \alpha^{1-\frac{z}{2}} \beta^{1-\frac{z}{2}}\right)
$$

is analytic. Here $\operatorname{tr}$ denotes the trace on the Haageup $L_{1}$ space. By the three lines lemma, we find

However, we have

$$
\left|\operatorname{tr}\left(c \alpha^{\eta} \beta^{\eta}\right)\right|=|h(\theta)| \leq\left(\sup _{z \in \partial_{0}}|h(z)|\right)^{1-\theta}\left(\sup _{z \in \partial_{1}}|h(z)|\right)^{\theta} .
$$

$$
\sup _{z \in \partial_{0}}|h(z)| \leq \sup _{z \in \partial_{0}}\|g(z)\|_{q^{\prime}}\left\|\alpha^{-z / 2} \alpha \beta \beta^{-z / 2}\right\|_{q} \leq \lambda,
$$

and

$$
\sup _{z \in \partial_{1}}|h(z)| \leq \sup _{z \in \partial_{1}}\|g(z)\|_{(2 q)^{\prime}}\left\|\alpha^{-\operatorname{Im}(z) / 2} \alpha^{\frac{1}{2}} \beta^{\frac{1}{2}} \beta^{-\operatorname{Im}(z) / 2}\right\|_{2 q} \leq \sqrt{\lambda} .
$$

Hence $\left|\operatorname{tr}\left(c \alpha^{\eta} \beta^{\eta}\right)\right| \leq \lambda^{1-\theta} \lambda^{\theta / 2}=\lambda^{\eta}$. Finally, we observe that our first argument for $\eta=1 / 2$ shows that if $\eta$ satisfies the assertion, then so does $\eta / 2$. Since the assertion holds for $1 / 2 \leq \eta \leq 1$, this completes the proof.

Theorem 3.2. If $2<p<\infty$ and $a, x \in L_{p}(N)_{+}$, we have

$$
\|a+x\|_{p}^{p}-\|a\|_{p}^{p} \leq p 2^{p-1} \max \left\{\left\|a^{p-1} x\right\|_{1},\|x\|_{p}^{p}\right\} .
$$


Proof. We begin by recalling Lemma 3.1 (part 1) of [Kos84b]. In this paper Kosaki used the uniform smoothness of $L_{p}(N)$ to show that the function $f(s)=\operatorname{tr}\left((a+s x)^{p}\right)$ is differentiable with derivative

$$
f^{\prime}(s)=p \operatorname{tr}\left((a+s x)^{p-1} x\right)
$$

This gives

$$
\operatorname{tr}\left((a+x)^{p}\right)-\operatorname{tr}\left(a^{p}\right)=p \int_{0}^{1} \operatorname{tr}\left((a+s x)^{p-1} x\right) d s .
$$

We define $k$ to be the natural number satisfying $k \leq p-1<k+1$ and define

$$
\theta=\frac{p-1-k}{p-1} \text { and }\left(\frac{1}{q}, \frac{1}{r}\right)=\left(\frac{k+1-\theta}{p}, \frac{p-k-1+\theta}{p}\right) .
$$

This implies $1 / r=\theta$ and $1 / q=1-\theta$. Then we may use Hölder's inequality and find

$$
\begin{aligned}
\operatorname{tr}\left((a+s x)^{p-1} x\right) & =\operatorname{tr}\left((a+s x)^{k} x^{1-\theta} x^{\theta}(a+s x)^{p-1-k}\right) \\
& \leq\left\|(a+s x)^{k} x^{1-\theta}\right\|_{q}\left\|x^{\theta}(a+s x)^{p-1-k}\right\|_{r} .
\end{aligned}
$$

By Lemma 3.1 for $(\alpha, \beta, \eta)=\left(x^{\frac{1}{p-1}}, a+s x, p-1-k\right)$, we get

$$
\left\|x^{\theta}(a+s x)^{p-1-k}\right\|_{r} \leq\left\|x^{\frac{1}{p-1}}(a+s x)\right\|_{p-1}^{p-1-k} .
$$

On the other hand, Lemma 3.1 for $(\alpha, \beta, \eta)=\left(x, a^{p-1}, 1 /(p-1)\right)$ gives

$$
\begin{aligned}
\left\|x^{\frac{1}{p-1}}(a+s x)\right\|_{p-1} & \leq\left\|x^{\frac{1}{p-1}} a\right\|_{p-1}+\left\|x^{\frac{p}{p-1}}\right\|_{p-1} \\
& \leq\left\|x a^{p-1}\right\|_{1}^{\frac{1}{p-1}}+\|x\|_{p}^{\frac{p}{p-1}} \leq 2 \max \left\{\left\|a^{p-1} x\right\|_{1},\|x\|_{p}^{p}\right\}^{\frac{1}{p-1}} .
\end{aligned}
$$

Let us set

$$
\xi=\max \left\{\left\|a^{p-1} x\right\|_{1},\|x\|_{p}^{p}\right\} .
$$

Then we find the following estimate for the second term on the right of (3.2)

$$
\left\|x^{\theta}(a+s x)^{p-1-k}\right\|_{r} \leq 2^{p-1-k} \xi^{\theta} .
$$

We now consider the first term. For a subset $A \subset\{1,2, \ldots, k\}$ we use the notation $a_{A^{c}} x_{A}=y_{1} \cdots y_{k}$ where $y_{i}=x$ if $i \in A$ and $y_{i}=a$ if $i \in A^{c}$. Then we deduce from the triangle inequality that

$$
\left\|(a+s x)^{k} x^{1-\theta}\right\|_{q} \leq \sum_{A} s^{|A|}\left\|a_{A^{c}} x_{A} x^{1-\theta}\right\|_{q} \leq \sum_{A}\left\|a_{A^{c}} x_{A} x^{1-\theta}\right\|_{q} .
$$

We claim that

$$
\left\|a_{A^{c}} x_{A} x^{1-\theta}\right\|_{q} \leq\left\|a^{p-1} x\right\|_{1}^{\frac{k-|A|}{p-1}}\|x\|_{p}^{1-\theta+|A|-\frac{k-|A|}{p-1}}
$$


Before proving our claim, let us show how to finish the argument

$$
\begin{aligned}
& \sum_{A}\left\|a_{A^{c}} x_{A} x^{1-\theta}\right\|_{q}=\sum_{j=0}^{k}\left(\begin{array}{c}
k \\
j
\end{array}\right)\left\|a^{p-1} x\right\|_{1}^{\frac{k-j}{p-1}}\|x\|_{p}^{1-\theta+j-\frac{k-j}{p-1}} \\
& \leq \sum_{j=0}^{k}\left(\begin{array}{c}
k \\
j
\end{array}\right) \xi^{\frac{(k-j)}{p-1}+\frac{1-\theta}{p}+\frac{j}{p}-\frac{k-j}{p(p-1)}}=\sum_{j=0}^{k}\left(\begin{array}{l}
k \\
j
\end{array}\right) \xi^{\frac{k}{p-1}+\frac{1-\theta}{p}-\frac{k}{p(p-1)}}=2^{k} \xi^{\frac{k+1-\theta}{p}}=2^{k} \xi^{1-\theta},
\end{aligned}
$$

where the last identity follows from $1-\theta=\frac{k}{p-1}$. The assertion then follows from the combination of (3.1, 3.2, 3.3) with the estimate given above. Therefore, it remains to prove our claim. We need to consider different cases. First assume that $A=\emptyset$, so that we have to prove (recall that $1-\theta=\frac{k}{p-1}$ ) the inequality $\left\|a^{k} x^{1-\theta}\right\|_{q} \leq\left\|a^{p-1} x\right\|_{1}^{1-\theta}$. This follows from Lemma 3.1 applied to $(\alpha, \beta, \eta)=\left(a^{p-1}, x, 1-\theta\right)$. Now assume $|A| \geq 1$. Then we may write

$$
a_{A^{c}} x_{A}=a^{\alpha_{1}} x^{\beta_{1}} a^{\alpha_{2}} \cdots x^{\beta_{r}} a^{\alpha_{r+1}}
$$

where $\sum_{i} \alpha_{i}+\sum_{i} \beta_{i}=k \leq p-1$. Since we have excluded the case $A=\emptyset$, all the coefficients $\alpha_{i}, \beta_{i}$ are strictly positive, except possibly $\alpha_{1}$ and $\alpha_{r+1}$. Let us first consider the case $\alpha_{1}>0=\alpha_{r+1}$. We define $q_{j}$ for $1 \leq j \leq r$ by $1 / q_{j}=\left(1+\alpha_{j}\right) / p$. Note that $1 \leq q_{j} \leq p$ for all $j$. Then we use Hölder's inequality and get

$$
\left\|a_{A^{c}} x_{A} x^{1-\theta}\right\|_{q} \leq\left(\prod_{j=1}^{r}\left\|a^{\alpha_{j}} x\right\|_{q_{j}}\|x\|_{p}^{\beta_{j}-1}\right)\|x\|_{p}^{1-\theta} .
$$

By Kosaki's interpolation theorem, we may estimate

$$
\left\|a^{\alpha_{j}} x\right\|_{q_{j}} \leq\|x\|_{p}^{1-\theta_{j}}\left\|a^{p-1} x\right\|_{1}^{\theta_{j}}
$$

where $\frac{1}{q_{j}}=\frac{1-\theta_{j}}{p}+\frac{\theta_{j}}{1}$. This means $\theta_{j}=\frac{\alpha_{j}}{p-1}$. Therefore we find

$$
\left\|a_{A^{c}} x_{A} x^{1-\theta}\right\|_{q} \leq\|x\|_{p}^{1-\theta} \prod_{j=1}^{r}\left\|a^{p-1} x\right\|_{1}^{\frac{\alpha_{j}}{p-1}}\|x\|_{p}^{\beta_{j}-\frac{\alpha_{j}}{p-1}}=\left\|a^{p-1} x\right\|_{1}^{\frac{k-|A|}{p-1}}\|x\|_{p}^{1-\theta+|A|-\frac{k-|A|}{p-1}} .
$$

This proves (3.4) for $\alpha_{1}>0=\alpha_{r+1}$. Let us now also assume that $\alpha_{1}=0$. Then we define the index $\widetilde{q}$ by $1 / \widetilde{q}=\left(\beta_{1}+\alpha_{2}+\beta_{2}\right) / p$. This allows us to apply Hölder's inequality as above and obtain

$$
\left\|a_{A^{c}} x_{A} x^{1-\theta}\right\|_{q} \leq\left\|x^{\beta_{1}} a^{\alpha_{2}} x^{\beta_{2}}\right\|_{\widetilde{q}}\left(\prod_{j=3}^{r}\left\|a^{\alpha_{j}} x\right\|_{q_{j}}\|x\|_{p}^{\beta_{j}-1}\right)\|x\|_{p}^{1-\theta} .
$$

We can assume without loss of generality (taking adjoints if necessary) that $\beta_{1} \leq \beta_{2}$. Define the index $\widehat{q}$ by $1 / \widehat{q}=\left(2 \beta_{1}+\alpha_{2}\right) / p$. Then we deduce the following estimate from 
Hölder's inequality and Lemma 3.1 applied to $(\alpha, \beta, \eta)=\left(x^{2 \beta_{1}}, a^{\alpha_{2}}, \frac{1}{2}\right)$

$$
\begin{aligned}
\left\|x^{\beta_{1}} a^{\alpha_{2}} x^{\beta_{2}}\right\|_{\widetilde{q}} & \leq\left\|x^{\beta_{1}} a^{\alpha_{2}} x^{\beta_{1}}\right\|_{\widehat{q}}\|x\|_{p}^{\beta_{2}-\beta_{1}}=\left\|x^{\beta_{1}} a^{\frac{\alpha_{2}}{2}}\right\|_{2 \widehat{q}}^{2}\|x\|_{p}^{\beta_{2}-\beta_{1}} \\
& \leq\left\|x^{2 \beta_{1}} a^{\alpha_{2}}\right\|_{\widehat{q}}\|x\|_{p}^{\beta_{2}-\beta_{1}} \leq\left\|x a^{\alpha_{2}}\right\|_{q_{2}}\|x\|_{p}^{\beta_{2}+\beta_{1}-1}=\prod_{1 \leq j \leq 2}\left\|a^{\alpha_{j}} x\right\|_{q_{j}}\|x\|_{p}^{\beta_{j}-1} .
\end{aligned}
$$

Therefore, the argument from above yields (3.4) in this case. Thus we have treated the cases $\alpha_{1}=0=\alpha_{r+1}$ and $\alpha_{1}>0=\alpha_{r+1}$. If $\alpha_{1}=0<\alpha_{r+1}$, we can take adjoints and use the same argument one more time. Let us now assume $\alpha_{1}>0$ and $\alpha_{r+1}>0$. If $\beta_{r} \geq 2$ the argument above applies by splitting $a^{\alpha_{r}} x^{\beta_{r}} a^{\alpha_{r+1}}=\left(a^{\alpha_{r}} x\right) x^{\beta_{r}-2}\left(x a^{\alpha_{r+1}}\right)$. Thus, the only case not covered so far is $\beta_{r}=1$. Here we have to use a little trick

$$
\left\|a^{\alpha_{r}} x a^{\alpha_{r+1}}\right\|_{\check{q}} \leq\left\|a^{\alpha_{r}+\alpha_{r+1}} x\right\|_{\check{q}} \quad \text { for } \quad 1 / \check{q}=\left(\alpha_{r}+\alpha_{r+1}+1\right) / p .
$$

Indeed, we define $d_{r}=a^{\alpha_{r}+\alpha_{r+1}}$ and $\gamma=\frac{\alpha_{r}}{\alpha_{r}+\alpha_{r+1}}$. Then

$$
\left\|a^{\alpha_{r}} x a^{\alpha_{r+1}}\right\|_{\check{q}}=\left\|d_{r}^{\gamma} x d_{r}^{1-\gamma}\right\|_{\check{q}} .
$$

Since the index $\check{q} \geq 1$, we may use complex interpolation and define the analytic function $f(z)=d_{r}^{z} x d_{r}^{1-z}$ on the strip. Then, the three lines lemma combined with the fact that $x$ is self-adjoint implies that

$$
\|f(\gamma)\|_{\check{q}} \leq \max \left\{\sup _{z \in \partial_{0}}\left\|d_{r}^{z} x d_{r}^{1-z}\right\|_{\check{q}}, \sup _{z \in \partial_{1}}\left\|d_{r}^{z} x d_{r}^{1-z}\right\|_{\check{q}}\right\} \leq \max \left\{\left\|x d_{r}\right\|_{\check{q}},\left\|d_{r} x\right\|_{\check{q}}\right\}=\left\|d_{r} x\right\|_{\check{q}} .
$$

This allows us to repeat the same argument and thereby completes the proof of (3.4).

Remark 3.3. If $N$ is commutative, the triangle inequality gives

$$
\begin{aligned}
\|a+x\|_{p}^{p}-\|a\|_{p}^{p} & =p \int_{0}^{1} \operatorname{tr}\left((a+s x)^{p-1} x\right) d s \\
& \leq p \int_{0}^{1}\left(\operatorname{tr}\left(a^{p-1} x\right)^{\frac{1}{p-1}}+\operatorname{str}\left(x^{p}\right)^{\frac{1}{p-1}}\right)^{p-1} d s \\
& \leq\left(2^{p}-1\right) \max \left\{\operatorname{tr}\left(a^{p-1} x\right), \operatorname{tr}\left(x^{p}\right)\right\} .
\end{aligned}
$$

However, in the noncommutative case it is known that the expression $\phi\left(|x|^{q}\right)^{1 / q}$ does not define a norm for arbitrary states. On the other hand, for $2 \leq p \leq 3$ Theorem 3.2 follows immediately from the fact that $t \mapsto t^{p-1}$ is operator convex. Indeed, we have

$$
(a+s x)^{p-1}=(1+s)^{p-1}\left(\frac{1}{1+s} a+\frac{s}{1+s} x\right)^{p-1} \leq(1+s)^{p-2}\left(a^{p-1}+s x^{p-1}\right) .
$$

This implies

$$
p \int_{0}^{1} \operatorname{tr}\left((a+s x)^{p-1} x\right) d s \leq \frac{p\left(2^{p-1}-1\right)}{p-1}\left(\operatorname{tr}\left(a^{p-1} x\right)+\operatorname{tr}\left(x^{p}\right)\right) .
$$


This is even better than our estimate.

Lemma 3.4. Let $d$ be the density of a normal state and consider the norm

$$
\|x\|_{p, t, d}=\max \left\{t^{\frac{1}{p}}\|x\|_{p}, t\left\|d^{\frac{1}{p^{\prime}}} x\right\|_{1}, t\left\|x d^{\frac{1}{p^{\prime}}}\right\|_{1}\right\} \quad \text { for } \quad 2 \leq p \leq \infty \quad \text { and } \quad t>0 .
$$

Then there are positive elements $x_{1}, x_{2}, x_{3}, x_{4}$ with $x=\sum_{k} i^{k} x_{k}$ and $\left\|x_{k}\right\|_{p, t, d} \leq\|x\|_{p, t, d}$.

Proof. Since $\left\|x^{*}\right\|_{p, t, d}=\|x\|_{p, t, d}$, we may clearly assume $x$ self-adjoint. For a self-adjoint element $x$, let $x_{+}=e_{+} x$ and $x_{-}=e_{-} x$ denote its positive and negative parts, where $e_{+}$and $e_{-}$stand for the corresponding spectral projections which commute with $x$. We recall from [Ter81] that $L_{q}(N)$ is a contractive $N$-bimodule for all $0<q<\infty$. Since $e_{+}$ commutes with $x$, we obtain

$$
\left\|e_{+} x\right\|_{p, t, d} \leq\|x\|_{p, t, d} .
$$

The same argument works for $x_{-}=e_{-} x$ and the assertion follows.

At the beginning of section 2 we defined the notion of a $(q,+)$-summing linear map $T: L_{p}(N) \rightarrow X$. Let $\pi_{q,+}(T)$ denote the infimum of all constants $c$ for which (2.1) holds. The following observation follows Pisier's argument in [Pis86a].

Lemma 3.5. Let $N$ be a von Neumann algebra. Let $T: L_{p}(N) \rightarrow X$ be a $(q,+)$-summing map with $(q,+)$-summing constant $\pi_{q,+}(T)$. Then, there exists a sequence $\left(a_{n}\right)$ of positive elements of norm 1 in $L_{p}(N)$ such that

$$
\lim _{n, \mathcal{U}}\left(1+\left\|\frac{T\left(x_{n}\right)}{\pi_{q,+}(T)}\right\|_{X}^{q}\right)^{\frac{1}{q}} \leq \lim _{n, \mathcal{U}}\left\|a_{n}+x_{n}\right\|_{p}
$$

holds for every bounded sequence $\left(x_{n}\right)$ in $L_{p}(N)_{+}$and every free ultrafilter $\mathcal{U}$.

Proof. Let $C_{n}$ be the smallest constant satisfying

$$
\left(\sum_{k=1}^{n}\left\|T\left(x_{k}\right)\right\|_{X}^{q}\right)^{\frac{1}{q}} \leq C_{n}\left\|\sum_{k=1}^{n} x_{k}\right\|_{p}
$$

for all families $\left(x_{1}, x_{2}, \ldots, x_{n}\right)$ in $L_{p}(N)_{+}$. In particular, we have $\pi_{q,+}(T)=\lim _{n} C_{n}$. Let $\left(\delta_{n}\right)$ be a sequence converging to 0 . Then we may find positive elements $y_{1}, y_{2}, \ldots, y_{n}$ in $L_{p}(N)$ such that

$$
\left(\sum_{k=1}^{n}\left\|T\left(y_{k}\right)\right\|_{X}^{q}\right)^{\frac{1}{q}}=1 \text { and }\left\|\sum_{k=1}^{n} y_{k}\right\|_{p} \leq C_{n}^{-1}\left(1+\delta_{n}\right) .
$$

Let $x_{n}$ be a positive element and set $y_{n+1}=x_{n}$, so that

$$
\left(1+\left\|T\left(x_{n}\right)\right\|_{X}^{q}\right)^{\frac{1}{q}}=\left(\sum_{k=1}^{n+1}\left\|T\left(y_{k}\right)\right\|_{X}^{q}\right)^{\frac{1}{q}} \leq C_{n+1}\left\|\left(\sum_{k=1}^{n} y_{k}\right)+x_{n}\right\|_{p}
$$




$$
\leq \frac{C_{n+1}}{C_{n}}\left(1+\delta_{n}\right)\left\|C_{n}\left(1+\delta_{n}\right)^{-1}\left(\sum_{k=1}^{n} y_{k}\right)+\pi_{q,+}(T) x_{n}\right\|_{p}
$$

Let us note that $\left\|C_{n}\left(1+\delta_{n}\right)^{-1} \sum_{k=1}^{n} y_{k}\right\|_{p} \leq 1$. Therefore, if we take

$$
a_{n}=\frac{C_{n}\left(1+\delta_{n}\right)^{-1} \sum_{k} y_{k}}{\left\|C_{n}\left(1+\delta_{n}\right)^{-1} \sum_{k} y_{k}\right\|_{p}},
$$

we obtain

$$
\left(1+\left\|T\left(x_{n}\right)\right\|_{X}^{q}\right)^{\frac{1}{q}} \leq\left(1+\delta_{n}\right) \frac{C_{n+1}}{C_{n}}\left\|a_{n}+\pi_{q,+}(T) x_{n}\right\|_{p} \quad \text { for all } n \geq 1 .
$$

Taking the limit yields the assertion.

Proposition 3.6. Let us fix $2 \leq q<p<\infty$. Given a von Neumann algebra $N$ and $a$ $(q,+)$-summing map $T: L_{p}(N) \rightarrow X$, there exists a sequence of densities $d_{n} \in L_{1}(N)$ with $\operatorname{tr}\left(d_{n}\right)=1$ such that

$$
\lim _{n, \mathcal{U}}\left\|T\left(x_{n}\right)\right\|_{X} \leq c(p, q) \pi_{q,+}(T) t^{-\frac{1}{q}} \lim _{n, \mathcal{U}}\left\|x_{n}\right\|_{p, t, d_{n}} .
$$

holds for all $t>0$ and bounded sequences $\left(x_{n}\right)$ in $L_{p}(N)$. In particular, we deduce

$$
\lim _{n, \mathcal{U}}\left\|T\left(x_{n}\right)\right\|_{X} \leq c(p, q) \pi_{q,+}(T) \lim _{n, \mathcal{U}}\left(\left\|x_{n}\right\|_{p}^{\frac{p^{\prime}}{q^{\prime}}} \max \left\{\left\|d_{n}^{\frac{1}{p^{\prime}}} x_{n}\right\|_{1},\left\|x_{n} d_{n}^{\frac{1}{p^{\prime}}}\right\|_{1}\right\}^{1-\frac{p^{\prime}}{q^{\prime}}}\right) .
$$

Proof. According to Lemma 3.4 and the linearity of $T$, we may clearly assume that the sequence $\left(x_{n}\right)$ lives in the positive cone $L_{p}(N)_{+}$. In particular, according to Lemma 3.5 we can find a sequence $\left(a_{n}\right)$ of norm 1 positive elements in $L_{p}(N)$ satisfying

$$
\lim _{n, \mathcal{U}}\left(1+\left\|\frac{T\left(x_{n}\right)}{\pi_{q,+}(T)}\right\|_{X}^{q}\right)^{p / q} \leq \lim _{n, \mathcal{U}}\left\|a_{n}+x_{n}\right\|_{p}^{p}
$$

Since $1+\alpha \lambda \leq(1+\lambda)^{\alpha}$ for $\lambda>0$ and $\alpha>1$, we deduce

$$
1+\frac{p}{q} \lim _{n, \mathcal{U}}\left\|\frac{T\left(x_{n}\right)}{\pi_{q,+}(T)}\right\|_{X}^{q} \leq \lim _{n, \mathcal{U}}\left\|a_{n}+x_{n}\right\|_{p}^{p}
$$

Recalling that $a_{n}$ is norm 1 in $L_{p}(N)$, we obtain by Theorem 3.2

$$
\begin{aligned}
\lim _{n, \mathcal{U}}\left\|T\left(x_{n}\right)\right\|_{X} & \leq \lim _{n, \mathcal{U}} \pi_{q,+}(T)\left(\frac{q}{p}\left(\left\|a_{n}+x_{n}\right\|_{p}^{p}-\left\|a_{n}\right\|_{p}^{p}\right)\right)^{\frac{1}{q}} \\
& \leq \lim _{n, \mathcal{U}} \pi_{q,+}(T)\left(q 2^{p-1} \max \left\{\left\|a_{n}^{p-1} x_{n}\right\|_{1},\left\|x_{n}\right\|_{p}^{p}\right\}\right)^{\frac{1}{q}} .
\end{aligned}
$$

We define $d_{n}=a_{n}^{p}$ and assume that $\left\|x_{n}\right\|_{p, t, d_{n}} \leq 1$. This implies that

$$
\max \left\{\left\|a_{n}^{p-1} x_{n}\right\|_{1},\left\|x_{n}\right\|_{p}^{p}\right\}=\frac{1}{t} \max \left\{\left(t^{\frac{1}{p}}\left\|x_{n}\right\|_{p}\right)^{p}, t\left\|d_{n}^{\frac{1}{p^{\prime}}} x_{n}\right\|_{1}, t\left\|x_{n} d_{n}^{\frac{1}{p^{\prime}}}\right\|_{1}\right\} \leq \frac{1}{t} .
$$


In conjunction with (3.5), this proves the first assertion for sequences $\left(x_{n}\right)$ of positive operators. A further constant 4 comes from Lemma 3.4 in the general case. Let us prove the second assertion. We define $\alpha=\lim _{n, \mathcal{U}}\left\|x_{n}\right\|_{p}$ and $\beta=\lim _{n, \mathcal{U}} \max \left\{\left\|d_{n}^{1 / p^{\prime}} x_{n}\right\|_{1},\left\|x_{n} d_{n}^{1 / p^{\prime}}\right\|_{1}\right\}$. By the first part we have

$$
\lim _{n, \mathcal{U}}\left\|T\left(x_{n}\right)\right\|_{X} \leq 4 c(p, q) \pi_{q,+}(T) \inf _{t>0} \max \left\{t^{\frac{1}{p}-\frac{1}{q}} \alpha, t^{1-\frac{1}{q}} \beta\right\} .
$$

The optimal choice is $t=\left(\frac{\alpha}{\beta}\right)^{p^{\prime}}$ and the optimal value is then given by

$$
\max \left\{t^{\frac{1}{p}-\frac{1}{q}} \alpha, t^{1-\frac{1}{q}} \beta\right\}=\alpha^{\frac{1-1 / q}{1-1 / p}} \beta^{\frac{1 / q-1 / p}{1-1 / p}}=\alpha^{p^{\prime} / q^{\prime}} \beta^{1-p^{\prime} / q^{\prime}} .
$$

Our next step through our Nikishin-type result requires some additional work and in particular the theory of ultraproducts, see Ray02, RX03] for some background. Let us assume that $N$ is a $\sigma$-finite von Neumann algebra and $d_{0}$ is a density of a normal faithful state $\phi_{0}$. We recall from Ray02 that

$$
\prod_{\mathcal{U}} L_{p}(N)=L_{p}\left(\left(\prod_{\mathcal{U}} N_{*}\right)^{*}\right)
$$

In the following we shall use the notation $\left(a_{n}\right)^{\bullet}$ for the canonical image of $\left(a_{n}\right)$ in the algebra $\left(\prod_{\mathcal{U}} N_{*}\right)^{*}$. Note that $\left\{\left(a_{n}\right)^{\bullet} \mid \sup _{n}\left\|a_{n}\right\|<\infty\right\}$ is dense in $\left(\prod_{\mathcal{U}} N_{*}\right)^{*}$ with respect to the strong operator topology. Following [RX03], we use the support $e$ of the ultraproduct state

$$
\phi_{\mathcal{U}}\left(\left(a_{n}\right)^{\bullet}\right)=\lim _{n, \mathcal{U}} \operatorname{tr}\left(d_{0} a_{n}\right) .
$$

Let us use the notation $N_{\mathcal{U}}=e\left(\prod_{\mathcal{U}} N_{*}\right)^{*} e$. Clearly, the state $\phi_{\mathcal{U}}$ is a normal faithful state on $N_{\mathcal{U}}$ and the space $L_{p}\left(N_{\mathcal{U}}\right)$ is canonically isomorphic to $e\left(\prod_{\mathcal{U}} L_{p}(N)\right) e$, see Ray02 for further details. This means we can represent elements $x$ in $L_{p}\left(N_{\mathcal{U}}\right)$ by sequences of the form $e\left(x_{n}\right)^{\bullet} e$. This applies in particular for $p=1$ and the representing sequence for $\phi_{\mathfrak{U}}$ is given by the constant sequence $\left(d_{0}\right)^{\bullet}$. Here and in the following we also use the notation $\left(x_{n}\right)^{\bullet}$ for the equivalence class in $\prod_{\mathcal{U}} L_{p}(N)$ of a bounded sequence $\left(x_{n}\right)$. Let us recall an observation from [RX03]. If $x \in L_{p}(N)$, then

$$
(1-e)(x)^{\bullet}=0 \text {. }
$$

Indeed, we may approximate $x$ by $d_{0}^{\frac{1}{p}} a_{n}$ with $a_{n} \in N$, so that

$$
(1-e)(x)^{\bullet}=(1-e)\left(d_{0}^{\frac{1}{p}} a_{n}\right)^{\bullet}=0
$$

because $e$ is the support of $\left(d_{0}\right)^{\bullet}$ and hence the support of its $p$-th root, see again Raynaud's paper Ray02 for more details on the Mazur map. Our aim is to replace the sequence of densities $\left(d_{n}\right)$ obtained in Proposition 3.6 by a single density $d$. 
Proposition 3.7. Let $2 \leq q<p<\infty$ and $\theta=1-\frac{p^{\prime}}{q^{\prime}}$. Given a $\sigma$-finite von Neumann algebra $N$ and a $(q,+)$-summing map $T: L_{p}(N) \rightarrow X$, there exists a density $\delta \in L_{1}\left(N_{\mathcal{U}}\right)$ of a normal faithful state $\phi_{\mathcal{U}}$ on $N_{\mathcal{U}}$ such that

$$
\lim _{n, \mathcal{U}}\left\|T\left(x_{n}\right)\right\|_{X} \leq c(p, q) \pi_{q,+}(T)\left\|\left(x_{n}\right)^{\bullet}\right\|_{\left[L_{p}\left(N_{\mathcal{U}}\right), \Delta_{p, 1}\left(\phi_{\mathcal{U}}\right)\right]_{\theta, 1}} .
$$

Moreover, if $1 \leq r<q$ and $\eta=\left(\frac{1}{q}-\frac{1}{p}\right) /\left(\frac{1}{r}-\frac{1}{p}\right)$, we also have

$$
\lim _{n, \mathcal{U}}\left\|T\left(x_{n}\right)\right\|_{X} \leq c(p, q) \pi_{q,+}(T)\left\|\left(x_{n}\right) \bullet\right\|_{\left[L_{p}\left(N_{\mathcal{U}}\right), \Delta_{p, r}\left(\phi_{\mathcal{U}}\right)\right]_{\eta, 1}} .
$$

Proof. For the first assertion, it suffices to show that

$$
\lim _{n, \mathcal{U}}\left\|T\left(x_{n}\right)\right\|_{X} \leq c(p, q) \pi_{q,+}(T)\left\|\left(x_{n}\right)^{\bullet}\right\|_{p}^{1-\theta}\left\|\left(x_{n}\right)^{\bullet}\right\|_{\Delta_{p, 1}\left(\phi_{\mathcal{U}}\right)}^{\theta}
$$

for a suitable density $\delta$ of a normal faithful state $\phi_{\mathcal{U}}$ in $N_{\mathcal{U}}$ and $\left(x_{n}\right)^{\bullet}$ in $L_{p}\left(N_{\mathcal{U}}\right)$. Indeed, this is a well-known property of the interpolation bracket $[,]_{\theta, 1}$, see e.g. [BL76, p.49]. On the other hand, according to Proposition 3.6 we have

$$
\lim _{n, \mathcal{U}}\left\|T\left(x_{n}\right)\right\|_{X} \leq c(p, q) \pi_{q,+}(T)\left\|\left(x_{n}\right)^{\bullet}\right\|_{p}^{1-\theta} \lim _{n, \mathcal{U}} \max \left\{\left\|d_{n}^{\frac{1}{p^{\prime}}} x_{n}\right\|_{1},\left\|x_{n} d_{n}^{\frac{1}{p^{\prime}}}\right\|_{1}\right\}^{\theta} .
$$

Therefore, it remains to find a normal faithful state $\phi_{\mathcal{U}}$ for which

$$
\lim _{n, \mathcal{U}} \max \left\{\left\|d_{n}^{\frac{1}{p^{\prime}}} x_{n}\right\|_{1},\left\|x_{n} d_{n}^{\frac{1}{p^{\prime}}}\right\|_{1}\right\} \leq c(p)\left\|\left(x_{n}\right)^{\bullet}\right\|_{\Delta_{p, 1}\left(\phi_{\mathcal{U}}\right)}
$$

whenever $\left(x_{n}\right)^{\bullet}$ belongs to $L_{p}\left(N_{\mathcal{U}}\right)$. We deduce for $\left(x_{n}\right) \in L_{p}\left(N_{\mathcal{U}}\right)$ that

$$
\lim _{n, \mathcal{U}}\left\|d_{n}^{\frac{1}{p^{\prime}}} x_{n}\right\|_{1}=\left\|\left(d_{n}^{\frac{1}{p^{\prime}}}\right)^{\bullet}\left(x_{n}\right)^{\bullet}\right\|_{1}=\left\|\left(d_{n}^{\frac{1}{p^{\prime}}}\right)^{\bullet} e\left(x_{n}\right)^{\bullet}\right\|_{1}=\left\|\left|\left(d_{n}^{\frac{1}{p^{\prime}}}\right)^{\bullet} e\right|\left(x_{n}\right)^{\bullet}\right\|_{1} \cdot
$$

Here we use the partial isometry between $\left(d_{n}^{\frac{1}{p^{\prime}}}\right)^{\bullet} e$ and $\left|\left(d_{n}^{\frac{1}{p^{\prime}}}\right)^{\bullet} e\right|$. Now, we define

$$
\delta_{0}=\left(\left|\left(d_{n}^{\frac{1}{p^{\prime}}}\right)^{\bullet} e\right|^{2 p^{\prime}}+\left(d_{0}^{2}\right)^{\bullet}\right)^{\frac{1}{2}} .
$$

Note that

$$
\left\|\delta_{0}\right\|_{1}=\left\|\delta_{0}^{2}\right\|_{\frac{1}{2}}^{\frac{1}{2}} \leq\left\|\left|\left(d_{n}^{\frac{1}{p^{\prime}}}\right)^{\bullet} e\right|^{2 p^{\prime}}\right\|_{\frac{1}{2}}^{\frac{1}{2}}+\left\|\left(d_{0}^{2}\right)^{\bullet}\right\|_{\frac{1}{2}}^{\frac{1}{2}}=\| \mid\left(d_{n}^{\frac{1}{p^{\prime}}} \bullet \bullet e \|_{p^{\prime}}^{p^{\prime}}+1 \leq 2 .\right.
$$

Thus, if we set $\delta=\delta_{0} /\left\|\delta_{0}\right\|_{1}$, we obtain the density of a normal faithful state on $N_{\mathcal{U}}$ given by $\phi_{\mathcal{U}}(\cdot)=\operatorname{tr}(\delta \cdot)$. Indeed, the normality is clear while the faithfulness follows from the fact that $\delta \geq \frac{1}{2}\left(d_{0}\right)^{\bullet}$, so that $\delta$ has full support. It is a state because $\|\delta\|_{1}=1$. We have

$$
\left|\left(d_{n}^{\frac{1}{p^{\prime}}}\right)^{\bullet} e\right|^{2 p^{\prime}} \leq \delta_{0}^{2} \Rightarrow\left|\left(d_{n}^{\frac{1}{p^{\prime}}}\right)^{\bullet} e\right| \leq \delta_{0}^{\frac{1}{p^{\prime}}}
$$

Hence we can find a contraction $w$ in $N_{\mathcal{U}}$ such that $\left|\left(d_{n}^{\frac{1}{p^{\prime}}}\right)^{\bullet} e\right|=w \delta_{0}^{\frac{1}{p^{\prime}}}=\delta_{0}^{\frac{1}{p^{\prime}}} w$. This implies

$$
\left\|\left|\left(d_{n}^{\frac{1}{p^{\prime}}}\right)^{\bullet} e\right|\left(x_{n}\right)^{\bullet}\right\|_{1}=\left\|w \delta_{0}^{\frac{1}{p^{\prime}}}\left(x_{n}\right)^{\bullet}\right\|_{1} \leq\left\|\delta_{0}^{\frac{1}{p^{\prime}}}\left(x_{n}\right)^{\bullet}\right\|_{1} \leq 2^{\frac{1}{p^{\prime}}}\left\|\delta^{\frac{1}{p^{\prime}}}\left(x_{n}\right)^{\bullet}\right\|_{1} .
$$


Similarly, we have

$$
\left\|\left(x_{n}\right)^{\bullet}\left(d_{n}^{\frac{1}{p^{\prime}}}\right)\right\|_{1}=\left\|\left(x_{n}\right)^{\bullet} e\left(d_{n}^{\frac{1}{p^{\prime}}}\right)\right\|_{1}=\left\|\left(x_{n}\right)^{\bullet}\left|\left(d_{n}^{\frac{1}{p^{\prime}}}\right)^{\bullet} e\right|\right\|_{1}=\left\|\left(x_{n}\right)^{\bullet} \delta_{0}^{\frac{1}{p^{\prime}}} w\right\|_{1} \leq 2^{\frac{1}{p^{\prime}}}\left\|\left(x_{n}\right)^{\bullet} \delta^{\frac{1}{p^{\prime}}}\right\|_{1}
$$

for all $\left(x_{n}\right) \in L_{p}\left(N_{\mathcal{U}}\right)$. Therefore, we obtain (3.6) and the first assertion is proved. The second assertion is an immediate consequence of the first one and the reiteration theorem. Indeed, according to Theorem [1.1 we have

$$
\Delta_{p, r}\left(\phi_{\mathcal{U}}\right)=\left[L_{p}\left(N_{\mathcal{U}}\right), \Delta_{p, 1}\left(\phi_{\mathcal{U}}\right)\right]_{\zeta}
$$

where $1 / r=\zeta+(1-\zeta) / p$, so that $\zeta=1-\frac{p^{\prime}}{r^{\prime}}$. The reiteration theorem for the real method [BL76, Theorem 4.7.2] implies $\left[L_{p}\left(N_{\mathcal{U}}\right), \Delta_{p, r}\left(\phi_{\mathcal{U}}\right)\right]_{\eta, 1}=\left[L_{p}\left(N_{\mathcal{U}}\right), \Delta_{p, 1}\left(\phi_{\mathcal{U}}\right)\right]_{\theta, 1}$ with $\theta=\eta \zeta$. We find $\eta=\left(\frac{1}{q}-\frac{1}{p}\right) /\left(\frac{1}{r}-\frac{1}{p}\right)$ as announced.

Corollary 3.8. Let $2<q<p<\infty$ and $\eta=\left(\frac{1}{q}-\frac{1}{p}\right) /\left(\frac{1}{2}-\frac{1}{p}\right)$. Given any von Neumann algebra $N$ and a $(q,+)$-summing map $T: L_{p}(N) \rightarrow X$, there exists a density $d \in L_{1}(N)$ with $\operatorname{tr}(d)=1$ and support e such that

$$
\|T(x)\|_{X} \leq c(p, q) \pi_{q,+}(T)\|x\|_{\left[e L_{p}(N)+L_{p}(N) e, \Delta_{p, 2}(\phi)\right]_{\eta, 1}} .
$$

Proof. Let us first assume that $N$ is a $\sigma$-finite von Neumann algebra and set $\frac{1}{r}=\frac{1}{2}-\frac{1}{p}$. We use the density $\delta=\left(\delta_{n}\right)^{\bullet} \in L_{1}\left(N_{\mathcal{U}}\right)$ from Proposition 3.7. Given $x \in L_{p}(N)$ we observe that we have

$$
\lim _{n, \mathcal{U}}\left\|\delta_{n}^{\frac{1}{r}} x\right\|_{2}^{2}=\lim _{n, \mathcal{U}} \operatorname{tr}\left(\delta_{n}^{\frac{1}{r}} x x^{*} \delta_{n}^{\frac{1}{r}}\right)=\lim _{n, \mathcal{U}} \operatorname{tr}\left(x x^{*} \delta_{n}^{\frac{2}{r}}\right)
$$

This defines a positive element $a=\lim _{n, \mathcal{U}} \delta_{n}^{\frac{2}{r}} \in L_{\frac{r}{2}}(N)$. We take $d=a^{\frac{r}{2}}$ and recall that

$$
\lim _{n, \mathcal{U}}\|x\|_{p}^{1-\eta} \max \left\{\left\|\delta_{n}^{\frac{1}{r}} x\right\|_{2},\left\|x \delta_{n}^{\frac{1}{r}}\right\|_{2}\right\}^{\eta}=\|x\|_{p}^{1-\eta} \max \left\{\left\|d^{\frac{1}{r}} x\right\|_{2},\left\|x d^{\frac{1}{r}}\right\|_{2}\right\}^{\eta} .
$$

Thus Proposition 3.7 applied to the constant sequence $(x)^{\bullet}$ yields the result, because $(x)^{\bullet}=e(x)^{\bullet} e$. When $N$ is an arbitrary von Neumann algebra, we choose a normal strictly semifinite weight $\psi=\lim _{i} \phi_{i}$ such that $\phi_{i}$ is a positive functional and the support $e_{i}$ of $\phi_{i}$ satisfies $\sigma_{t}^{\phi_{i}}\left(e_{i}\right)=e_{i}$. Then $N_{i}=e_{i} N e_{i}$ is $\sigma$-finite and we find a density $d_{i} \in L_{1}\left(N_{i}\right)$ with $\operatorname{tr}\left(d_{i}\right)=1$ and such that

$$
\left\|T\left(e_{i} x e_{i}\right)\right\|_{X} \leq c(p, q) \pi_{q,+}(T)\left\|e_{i} x e_{i}\right\|_{p}^{1-\eta} \max \left\{\left\|d_{i}^{\frac{1}{r}} x\right\|_{2},\left\|x d_{i}^{\frac{1}{r}}\right\|_{2}\right\}^{\eta} .
$$

As above we can pass to the limit $d^{\frac{2}{r}}=\lim _{i} d_{i}^{\frac{2}{r}}$. 
Remark 3.9. It is tempting to use a weak limit $d=\lim _{n, \mathcal{U}} d_{n}^{1 / p^{\prime}} \in L_{p^{\prime}}(N)$ in Proposition 3.6. The problem we face is the equality

$$
\|d x\|_{1} \stackrel{?}{=} \lim _{n, \mathcal{U}}\left\|d_{n}^{1 / p^{\prime}} x\right\|_{1} .
$$

This equality does not hold in general. Indeed, assuming (3.7), we would deduce from the polar decomposition that $\left\|w^{*}-\lim _{n, \mathcal{U}} a_{n} x\right\|_{1}=\lim _{n, \mathcal{U}}\left\|a_{n} x\right\|_{1}$ holds for all bounded sequences $a_{n}$ and $x \in S_{p}=L_{p}\left(B\left(\ell_{2}\right), t r\right)$. In $S_{p^{\prime}}$ we may choose $a_{n}=e_{n, 1}$. Then $\lim _{n} a_{n}=0$ weakly and $\lim _{n}\left\|a_{n} e_{11}\right\|_{1}=1$. We suspect that we need some equi-integrability for (3.7) to hold. Our proof does not provide any equi-integrability condition.

We are ready for the main result.

Theorem 3.10. Let $2 \leq q<p<\infty$ and let $N$ be any von Neumann algebra. Given $a(q,+)$-summing map $T: L_{p}(N) \rightarrow X$, there exists a density $d \in L_{1}(N)$ such that the inequality below holds for any index $q<r<p$

$$
\|T(x)\|_{X} \leq c(p, q, r) \pi_{q,+}(T)\|x\|_{\Delta_{p, r}(\phi)} .
$$

Moreover, there exists a map $\widetilde{T}: L_{r}(N \oplus N) \rightarrow X$ such that

$$
T(x)=\widetilde{T}\left(d^{\frac{1}{r}-\frac{1}{p}} x, x d^{\frac{1}{r}-\frac{1}{p}} x\right)=\widetilde{T}\left(j_{p, r}(x)\right) \quad \text { and } \quad\|\widetilde{T}\| \leq c(p, q, r) \pi_{q,+}(T) .
$$

Proof. According to Corollary 3.8, we may find $d$ such that

$$
\|T(x)\|_{X} \leq c(p, q) \pi_{q,+}(T)\|x\|_{p}^{1-\eta} \max \left\{\left\|d^{\frac{1}{2}-\frac{1}{p}} x\right\|_{2},\left\|x d^{\frac{1}{2}-\frac{1}{p}}\right\|_{2}\right\}^{\eta} .
$$

Let $e$ denote the support of $d$ and let $\phi(x)=\operatorname{tr}(d x)$ be the associated state. Let us decompose any element $x$ in $L_{p}(N)$ as $x=e x e+e x(1-e)+(1-e) x e+(1-e) x(1-e)$. Then we note that $T$ vanishes on the corner $(1-e) L_{p}(N)(1-e)$. We apply Lemma 2.1 for $\tilde{\eta}<\eta$ and deduce

$$
\left.\|T(x)\|_{X} \leq c(p, q) c(\eta, \tilde{\eta}) \pi_{q,+}(T)\|x\|_{\left[e L_{p}(N)+L_{p}(N) e, \Delta_{p, 2}(\phi)\right.}\right]_{\tilde{\eta}}
$$

for all $x \in L_{p}(N)$ such that $(1-e) x(1-e)=0$. We recall the isomorphism from Lemma 2.2 ;

$$
\left[e L_{p}(N)+L_{p}(N) e, \Delta_{p, 2}(\phi)\right]_{\tilde{\eta}} \simeq e \Delta_{p, r}(\phi) e \oplus e L_{r}(N)(1-e) \oplus(1-e) L_{r}(N) e,
$$

where $\frac{1}{r}=\frac{1-\tilde{\eta}}{p}+\frac{\tilde{\eta}}{2}=\frac{1}{p}+\tilde{\eta}\left(\frac{1}{2}-\frac{1}{p}\right)<\frac{1}{p}+\eta\left(\frac{1}{2}-\frac{1}{p}\right)=\frac{1}{q}$. Thus for every $q<r<p$ we can find a suitable $\tilde{\eta}$. Note that $\eta=1$ when $q=2$, but then we may use that $(2,+)$-summing implies $(q,+)$-summing for all $q>2$. We denote by

$$
T_{\tilde{\eta}}: e \Delta_{p, r}(\phi) e \oplus e L_{r}(N)(1-e) \oplus(1-e) L_{r}(N) e \rightarrow X
$$


the corresponding bounded map given by (3.8) and (3.9). Now we proceed as in Theorem 2.3 and define

$$
\widetilde{\mathcal{Q}}_{r}: L_{r}(N) \oplus L_{r}(N) \rightarrow e \Delta_{p, r}(\phi) e \oplus e L_{r}(N)(1-e) \oplus(1-e) L_{r}(N) e
$$

by $\widetilde{\mathcal{Q}}_{r}(x, y)=\mathcal{Q}_{r}($ exe, eye $) \oplus \operatorname{ex}(1-e) \oplus(1-e) y e$, where $\mathcal{Q}_{r}$ is the projection from Theorem 1.1. This allows us to define $\widetilde{T}(x, y)=T_{\tilde{\eta}} \widetilde{\mathcal{Q}}_{r}$ so that

$$
\begin{aligned}
\widetilde{T}\left(d^{\frac{1}{r}-\frac{1}{p}} x, x d^{\frac{1}{r}-\frac{1}{p}}\right) & =T_{\tilde{\eta}}\left((\text { exe }, \text { exe }) \oplus d^{\frac{1}{r}-\frac{1}{p}} x(1-e) \oplus(1-e) x d^{\frac{1}{r}-\frac{1}{p}}\right) \\
& =T(e x e+(1-e) x+e x(1-e))=T(x) .
\end{aligned}
$$

The norm estimate follows from Theorem 1.1, (3.8) and (3.9) (see Lemma 2.2).

Remark 3.11. In the commutative analog of Theorem 3.10 , the restriction $p, q \geq 2$ is not needed. It would be very interesting to know whether this restriction is necessary in the noncommutative setting.

Remark 3.12. We know from Lust-Piquard work LP92 that for $q=2$ the situation is much nicer. Let $\frac{1}{q}+\frac{1}{s}=\frac{1}{2}$. Then, the noncommutative Khintchine inequality [LP86] implies

$$
\sum_{k}\left\|T\left(x_{k}\right)\right\|_{X}^{2} \leq c_{p}^{2} c_{2}(T) \sup _{\|a\|_{s / 2} \leq 1} \sum_{k} \operatorname{tr}\left(a\left(x_{k}^{*} x_{k}+x_{k} x_{k}^{*}\right)\right) .
$$

Applying the standard separation argument one obtains a factorization $T=v j_{p, 2}$ through the inclusion map $i d: L_{p}(N) \rightarrow \Delta_{p, 2}(\phi)$. We refer to [LP92] for more details and to [LPX] for further information.

Corollary 3.13. Let $1<q<r \leq 2$ and $T: X \rightarrow L_{q}(N)$ be a linear map such that $T^{*}$ has cotype $r^{\prime}$. Then there exists a density $d \in L_{1}(N)$ with $\operatorname{tr}(d)=1$ such that for every $q<p<r$ there exists a map $u: X \rightarrow L_{p}(N)$ satisfying

$$
T(x)=d^{\frac{1}{q}-\frac{1}{p}} u(x)+u(x) d^{\frac{1}{q}-\frac{1}{p}} \quad \text { for all } \quad x \in X .
$$

Proof. By Theorem 3.10 we find a density $d$ with $\operatorname{tr}(d)=1$ such that

$$
\left\|T^{*}: \Delta_{q^{\prime}, p^{\prime}}(\phi) \rightarrow X^{*}\right\| \leq c\left(p^{\prime}, q^{\prime}, r^{\prime}\right) \pi_{r^{\prime},+}\left(T^{*}\right) \leq 2 c\left(p^{\prime}, q^{\prime}, r^{\prime}\right) c_{r^{\prime}}\left(T^{*}\right)
$$

for every $r^{\prime}<p^{\prime}<q^{\prime}$. We write $T_{q^{\prime}, p^{\prime}}^{*}: \Delta_{q^{\prime}, p^{\prime}}(\phi) \rightarrow X^{*}$ for the corresponding map. Let $\widetilde{\mathcal{Q}}_{p^{\prime}}$ : $L_{p^{\prime}}(N) \rightarrow \Delta_{q^{\prime}, p^{\prime}}(\phi)$ be the projection from Theorem 3.10. We recall that $\mathcal{Q}_{p^{\prime}}^{*}=\left(v_{e}, v_{e}\right)$ has two identical components. As in the proof of Theorem 2.3 we define $v: \Delta_{q^{\prime}, p^{\prime}}(\phi)^{*} \rightarrow L_{p}(N)$ by $v\left(\xi_{e}, \xi_{1-e, e}, \xi_{e, 1-e}\right)=v_{e}\left(\xi_{e}\right)+\xi_{1-e, e}+\xi_{e, 1-e}$. Following the argument from Theorem 2.3 we can check that $u=v\left(T_{q^{\prime}, p^{\prime}}^{*}\right)^{*}: X \rightarrow L_{p}(N)$ provides the corresponding decomposition. Note that in the $\sigma$-finite case we may assume that $d$ has full support. Then formally

$$
u(x)=\left(\mathcal{L}_{d^{\frac{1}{q}-\frac{1}{p}}}+\mathcal{R}_{d^{\frac{1}{q}-\frac{1}{p}}}\right)^{-1} T(x) .
$$


In full generality we have

$$
u(x)=\left(\mathcal{L}_{d^{\frac{1}{q}-\frac{1}{p}}}+\mathcal{R}_{d^{\frac{1}{q}-\frac{1}{p}}}\right)^{-1} e T(x) e+d^{\frac{1}{p}-\frac{1}{q}} T(x)(1-e)+(1-e) T(x) d^{\frac{1}{p}-\frac{1}{q}} .
$$

We should warn the reader that these multiplications are usually not well-defined, see both Step 2 and Step 3 of the proof of Theorem 1.1 for a rigorous interpretation using Haagerup's construction.

Proof of Theorem $\mathrm{B}$. Let $1 \leq q<2$ and let $X$ be an infinite-dimensional subspace of $L_{q}(N)$ not containing $\ell_{q}$. According to Raynaud and Xu's result [RX03, Theorem5.1] we deduce that $X$ does not contain $\ell_{q}(n)$ 's uniformly. By the Krivine-Maurey-Pisier theorem, the type index of $X$ satisfies $p_{X}>q$. Let $q<r<p_{X}$ so that $X^{*}$ has cotype $r^{\prime}$. Let $\iota: X \rightarrow L_{q}(N)$ be the inclusion map. Then $T=\iota^{*}: L_{q^{\prime}}(N) \rightarrow X^{*}$ has cotype $r^{\prime}$ and the assertion follows from Corollary 3.13 .

We refer to [Ran02, RX03] for the definition of $q$-equiintegrable sets in $L_{q}(N)$.

Corollary 3.14. If $X \subset L_{q}(N)$ and $1 \leq q<2$, the following are equivalent

i) The unit ball of $X$ is q-equiintegrable.

ii) There exists a density $d \in L_{1}(N)$ such that

$$
u: x \in X \mapsto\left(d^{\frac{1}{r}-\frac{1}{q}} x, x d^{\frac{1}{r}-\frac{1}{q}}\right) \in L_{r}(N \oplus N)
$$

is an isomorphic embedding for some (all) $0<r<q$.

iii) There exists $q<p<2$ and a bounded linear map

$$
u: X \rightarrow L_{p}(N)
$$

such that $x=d^{\frac{1}{q}-\frac{1}{p}} u(x)+u(x) d^{\frac{1}{q}-\frac{1}{p}}$ for some positive density $d \in L_{1}(N)$.

Proof. According to [RX03, Theorem 5.1], the conditions i) and ii) are both equivalent to the fact that $X$ does not contain $\ell_{q}$ and hence imply iii) by means of Theorem $\mathrm{B}$. On the other hand, iii) implies that $X$ has type $p>q$ and hence can not contain $\ell_{q}$.

Acknowledgement. The referee's comments of the first version of this paper not only improved the presentation, but enabled us to simplify the results considerably. We are also indebted to him for allowing us to present his proof of Theorem 1.15, We would like to thank Narcisse Randrianantoanina for keeping us up to date on his closely related work. The first-named author was partially supported by the National Science Foundation, DMS-0301116 and DMS 05-56120. The second-named author was partially supported by "Programa Ramón y Cajal, 2005" and the Grant MTM2004-00678, Spain. 


\section{REFERENCES}

[Ald81] D. J. Aldous. Subspaces of $L^{1}$, via random measures. Trans. Amer. Math. Soc. 267 (1981), 445-463.

[Ara90] H. Araki. On an inequality of Lieb and Thirring. Lett. Math. Phys. 19 (1990), 167-170.

[BL76] J. Bergh and J. Löfström. Interpolation spaces. An Introduction. Grundlehren der Mathematischen Wissenschaften 223. Springer-Verlag, 1976.

[BGM86] E. Berkson, T. A. Gillespie, and P. S. Muhly. Abstract spectral decompositions guaranteed by the Hilbert transform. Proc. London Math. Soc. (3) 53 (1986), 489-517.

[Bou86] J. Bourgain. Vector-valued singular integrals and the $H^{1}-B M O$ duality. Probability theory and harmonic analysis (Cleveland, Ohio, 1983), Monogr. Textbooks Pure Appl. Math. 98, 1-19. Dekker, New York, 1986.

[Fac87] T. Fack. Type and cotype inequalities for noncommutative $L^{p}$-spaces. J. Operator Theory 17 (1987), 255-279.

[FK86] T. Fack and H. Kosaki. Generalized s-numbers of $\tau$-measurable operators. Pacific J. Math. 123 (1986), 269-300.

[GK69] I. C. Gohberg and M. G. Krein. Introduction to the theory of linear nonselfadjoint operators. Transl. Math. Monogr. 18. American Mathematical Society, Providence, 1969.

[Haa79] U. Haagerup. $L^{p}$-spaces associated with an arbitrary von Neumann algebra. Algèbres d'opérateurs et leurs applications en physique mathématique, 175-184. CNRS, Paris, 1979.

[Hil81] M. Hilsum. Les espaces $L^{p}$ d'une algèbre de von Neumann définies par la derivée spatiale. J. Funct. Anal. 40 (1981), 151-169.

[Jun02] M. Junge. Doob's inequality for non-commutative martingales. J. Reine Angew. Math. 549 (2002), 149-190.

[Jun04] M. Junge. Fubini's theorem for ultraproducts of noncommmutative $L_{p}$-spaces. Canad. J. Math. 56 (2004), 983-1021.

[JLX03] M. Junge, C. Le Merdy and Q. Xu. Calcul fonctionnel et fonctions carrées dans les espaces $L^{p}$ non commutatifs. C. R. Math. Acad. Sci. Paris 337 (2003), 93-98.

[JP05] M. Junge and J. Parcet. Theory of amalgamated $L_{p}$ spaces in noncommutative probability. Preprint 2005, math.OA/0511406

[JR] M. Junge and H. Rosenthal. Noncommutative $L_{p}$ spaces are asymptotially stable. In progress.

[JX03] M. Junge and Q. Xu. Noncommutative Burkholder/Rosenthal inequalities. Ann. Probab. 31 (2003), 948-995.

[JXa] M. Junge and Q. Xu. Noncomutative Maximal Ergodic Theorems. Preprint 2005, math.OA/0505308. To appear in J. Amer. Math. Soc.

[JXb] M. Junge and Q. Xu. Haagerup's reduction on noncommutative $L_{p}$-spaces and aplications. In progress.

[KLW92] V. Kaftal, D. Larson and G. Weiss. Quasitriangular subalgebras of semifinite von Neumann algebras are closed J. Funct. Anal. 107 (1992), 387-401.

[KP62] M. I. Kadec and A. Pełczyński. Bases, lacunary sequences and complemented subspaces in the spaces $L_{p}$. Studia Math. 21 (1961/62), 161-176.

[KR97a] R. V. Kadison and J. R. Ringrose. Fundamentals of the theory of operator algebras I. Graduate Studies in Mathematics 15. American Mathematical Society, Providence, 1997. 
[KR97b] R. V. Kadison and J. R. Ringrose. Fundamentals of the theory of operator algebras II. Graduate Studies in Mathematics 16. American Mathematical Society, Providence, 1997.

[Kos84a] H. Kosaki. Applications of the complex interpolation method to a von Neumann algebra: noncommutative $L^{p}$-spaces. J. Funct. Anal. 56 (1984), 29-78.

[Kos84b] H. Kosaki. Applications of uniform convexity of noncommutative $L^{p}$-spaces. Trans. Amer. Math. Soc. 283 (1984), 265-282.

[Kos92] H. Kosaki. An inequality of Araki-Lieb-Thirring (von Neumann algebra case). Proc. Amer. Math. Soc. 114 (1992), 477-481.

[KM81] J. L. Krivine and B. Maurey. Espaces de Banach stables. Israel J. Math. 39 (1981), 273-295.

[KP70] S. Kwapień and A. Pełczyński. The main triangle projection in matrix spaces and its applications. Studia Math. 34 (1970), 43-68.

[LP86] F. Lust-Piquard. Inégalités de Khintchine dans $C_{p}(1<p<\infty)$. C. R. Acad. Sci. Paris Sér. I Math. 303 (1986), 289-292.

[LP92] F. Lust-Piquard. A Grothendieck factorization theorem on 2-convex Schatten spaces. Israel J. Math. 79 (1992), 331-365.

[LPX] F. Lust-Piquard and Q. Xu. The little Grothendieck theorem and Khintchine inequalities for symmetric spaces of measurable operators. Preprint 2006, math.FA/0609356. To appear in J. Funct. Anal.

[MN97] J. L. Marcolino Nhany. La stabilité des espaces L ${ }^{p}$ non-commutatifs. Math. Scand. 81 (1997), $212-218$.

[MS86] V. D. Milman and G. Schechtman. Asymptotic theory of finite-dimensional normed spaces. Lecture Notes in Math. 1200. Springer-Verlag, Berlin, 1986.

[Nel74] E. Nelson. Notes on non-commutative integration. J. Funct. Anal. 15 (1974), 103-116.

[Pis86a] G. Pisier. Factorization of linear operators and geometry of Banach spaces. CBMS Regional Conference Series in Mathematics 60, 1986.

[Pis86b] G. Pisier. Factorization of operators through $L_{p \infty}$ or $L_{p 1}$ and noncommutative generalizations. Math. Ann. 276 (1986), 105-136.

[Pis86c] G. Pisier. Probabilistic methods in the geometry of Banach spaces. Probability and analysis (Varenna, 1985). Lecture Notes in Math. 1206, 167-241. Springer, Berlin, 1986.

[Pis92] G. Pisier. Interpolation between $H^{p}$ spaces and noncommutative generalizations I. Pacific J. Math. 155 (1992), 341-368.

[Pis93] G. Pisier. Interpolation between $H^{p}$ spaces and noncommutative generalizations II. Rev. Mat. Iberoamericana 9 (1993), 281-291.

[Pis04] G. Pisier. The operator Hilbert space $O H$ and type III von Neumann algebras. Bull. London Math. Soc. 36 (2004), 455-459.

[PX97] G. Pisier and Q. Xu. Non-commutative martingale inequalities. Comm. Math. Phys. 189 (1987), 667-698.

[PX03] G. Pisier and Q. Xu. Non-commutative $L^{p}$-spaces. Handbook of the geometry of Banach spaces 2, 1459-1517. North-Holland, Amsterdam, 2003.

[Ran02] N. Randrianantoanina. Kadec-Petczyński decomposition for Haagerup $L^{p}$-spaces. Math. Proc. Cambridge Philos. Soc. 132 (2002), 137-154.

[Ray02] Y. Raynaud. On ultrapowers of non commutative $L_{p}$ spaces. J. Operator Theory 48 (2002), 41-68. 
[RX03] Y. Raynaud and Q. Xu. On subspaces of non-commutative $L_{p}$-spaces. J. Funct. Anal. 203 (2003), 149-196.

[Ros73] H. P. Rosenthal. On subspaces of $L^{p}$. Ann. of Math. 97 (1973), 344-373.

[Tak79] M. Takesaki. Theory of operator algebras I. Springer-Verlag, New York, 1979.

[Tak03a] M. Takesaki. Theory of operator algebras II. Encyclopaedia of Mathematical Sciences 125. Operator Algebras and Non-commutative Geometry 6. Springer-Verlag, Berlin, 2003.

[Tak03b] M. Takesaki. Theory of operator algebras III. Encyclopaedia of Mathematical Sciences 127. Operator Algebras and Non-commutative Geometry 8. Springer-Verlag, Berlin, 2003.

[Ter81] M. Terp. $L_{p}$ spaces associated with von Neumann algebras. Notes. Math. Institute, Copenhagen Univ., 1981.

[Ter82] M. Terp. Interpolation spaces between a von Neumann algebra and its predual. J. Operator Theory 8 (1982), 327-360.

[TJ84] N. Tomczak-Jaegermann. Uniform convexity of unitary ideals. Israel J. Math. 48 (1984), 249254 .

2000 Mathematics Subject Classification: Primary 46L53, Secondary 46B25.

Keyword's: Rosenthal's theorem, noncommutative $L_{p}$ spaces.

\section{Marius Junge}

Department of Mathematics

UNIVERSITY OF ILLINOIS AT URBANA-CHAMPAIGN

273 Altgeld Hall, 1409 W. Green Street, Urbana, IL 61801, USA

junge@math.uiuc.edu

Javier Parcet

DEPARTAMENTo DE MATEMÁticas

Instituto de Matemáticas y Física Fundamental Consejo Superior de Investigaciones Científicas

Depto de Matemáticas, Univ. Autónoma de Madrid, 28049, Spain

javier.parcet@uam.es 